\title{
An intratumoral injectable, electrostatic, cross-linkable curcumin depot and synergistic enhancement of anticancer activity
}

\author{
Seung Hun Park ${ }^{1}$, Da Yeon Kim ${ }^{1}$, Prasamsha Panta ${ }^{1}$, Ji Yeon Heo, Hye Yun Lee, Jae Ho Kim, \\ Byoung Hyun Min and Moon Suk Kim
}

In this study, we describe an intratumoral injectable, electrostatic, cross-linkable curcumin (Cur) drug depot to enhance anticancer activity. The key concept in this work was the preparation of an electrostatic, cross-linked carboxymethyl cellulose (CMC) and chitosan (CHI) hydrogel containing Cur-loaded microcapsules (Cur-M). The $\mathrm{CMC}$ and $\mathrm{CHI}$ solutions existed as a liquid before mixing and formed a $\mathrm{CMC}$ and $\mathrm{CHI}(\mathrm{CCH})$ hydrogel as a drug depot after mixing via electrostatic interactions between the anionic $\mathrm{CMC}$ and cationic $\mathrm{CHI}$. Compared with the individual $\mathrm{CMC}$ and $\mathrm{CHI}$ solutions, the electrostatic, cross-linked $\mathrm{CCH}$ depot persisted in vivo for an extended period. The prepared Cur-M was easily mixed with the $\mathrm{CMC}$ and $\mathrm{CHI}$ solutions. Cur-M/CMC and Cur-; M/CHI solutions easily formed Cur-M-loaded CCH depots after simple mixing. The in vitro and in vivo Cur-M-loaded CCH depot was designed with Cur-M dispersed inside an outer shell of electrostatically cross-linked $\mathrm{CCH}$. The Cur-M-loaded $\mathrm{CCH}$ depot produced greater inhibition of tumor growth than did Cur-M, whereas single and repeated injections of free Cur had the weakest inhibitory effects. The results of this study indicate that the electrostatic, cross-linked, Cur-M-loaded CCH depot described in this study can synergistically enhance anticancer activity in chemotherapeutic delivery systems.

NPG Asia Materials (2017) 9, e397; doi:10.1038/am.2017.102; published online 30 June 2017

\section{INTRODUCTION}

Injectable hydrogels have been utilized in diverse biomedical applications for a long time. ${ }^{1}$ These injectable materials can form hydrogels in physiological environments. Various biological factors can be incorporated easily and quantitatively into the injectable hydrogel solution by simple stirring in the liquid state. In addition, hydrogels are easy to handle and minimally invasive at target sites in the form of simple aqueous state injections and can replace traditional surgical procedures.

Various biomaterials are the subject of ongoing research to develop injectable hydrogels. ${ }^{2-7}$ An ionized biomaterial that bears cationic or anionic charges can exist in solution in a homogeneous state. ${ }^{8}$ However, if this biomaterial solution is mixed with another that possesses an opposite charge, electrostatic interactions can result in the formation of electrostatic cross-linking between the biomaterials.

There are several biomaterials, including carboxymethyl cellulose (CMC) and chitosan (CHI). CMC has numerous carboxylate groups that exhibit anionic polyelectrolyte properties with a zeta potential of approximately $-25 \mathrm{mV} .{ }^{9} \mathrm{CHI}$ possesses numerous primary aliphatic amine groups that can be solubilized by protonation under acidic conditions. Protonated $\mathrm{CHI}$ exhibits cationic polyelectrolyte properties with a zeta potential of approximately $+50 \mathrm{mV} .{ }^{10}$ Mixing of CMC and $\mathrm{CHI}$ leads to rapid formation of cross-linking via electrostatic interaction with oppositely charged polyelectrolytes, without catalyst or external energy, under physiological conditions and in aqueous media.

Thus, the primary goal of this work was to prepare an electrostatic, cross-linkable $\mathrm{CMC}$ and $\mathrm{CHI}$ formulation that is capable of forming a $\mathrm{CMC}$ and $\mathrm{CHI}$ drug depot $(\mathrm{CCH})$ in vivo, and to elucidate its in vivo applicability. In addition, we prepared CHI-fluorescein isothiocyanate (FITC) and CMC-near-infrared (NIR) to assess the in vivo fate of the electrostatically cross-linked depots over an extended period.

Currently available cancer drugs have had success in systemic chemotherapy. However, several problems are associated with cancer drug therapy, such as high cost, poor distribution to the target tumor due to rapid drug clearance and dose-limiting toxic side effects resulting from nonspecific distribution to healthy normal tissues. ${ }^{11}$

Curcumin (Cur) is easily and economically available from plant extracts or chemical synthesis. ${ }^{12}$ Moreover, Cur was shown to exhibit activities against numerous cancers in human clinical trials and is safe and well tolerated in cancer patients even at high doses. ${ }^{13}$

Although Cur has shown efficacy against numerous cancers, its therapeutic potential has been limited by poor bioavailability due to poor absorption, rapid metabolism and rapid systemic elimination. ${ }^{14-16}$ Therefore, research has been focused on improving the limitations and drawbacks of the conventional delivery of Cur. 
Microencapsulation technologies have advanced significantly during the past few decades as suitable carriers for long-term drug delivery, leading to many successful commercial products. ${ }^{15-19}$ Thus, a sustained Cur drug-delivery system using a microcapsule depot can offer potential clinical advantages, such as significantly reduced dosage frequency and improved efficacy without toxicity.

Intratumoral injection of anticancer drugs can achieve a high local concentration of the anticancer drug at the target tumor site. ${ }^{20-24}$ Such targeted direct delivery of the anticancer drug to the target can improve drug efficacy, reduce systemic drug concentration, and decrease the incidence of side effects compared with traditional chemotherapeutic drug administrations.

Therefore, we hypothesized that a Cur-loaded microcapsule (Cur$\mathrm{M})$, in which Cur is encapsulated in the inner core and surrounded by a poly(D,L-lactic-co-glycolic acid) (PLGA) shell, is expected to be a good candidate for improving the bioavailability of Cur through sustaining release of Cur ranging from weeks to months. Thus, as the second goal of this work, we prepared Cur-M as a candidate for sustained Cur drug delivery.

The electrostatic, cross-linkable CMC and $\mathrm{CHI}$ formulation with Cur-M is expected to be easily injected through a syringe needle. Mixing of the $\mathrm{CMC}$ and $\mathrm{CHI}$ solutions with the Cur-M was the easiest method to prepare the injectable, electrostatic, cross-linkable formulation. We hypothesized that a combination of electrostatic, crosslinkable CMC and CHI formulation, and Cur-M is an effective formulation for intratumoral injection. Thus, as the third goal of this work, the Cur-M-loaded $\mathrm{CCH}$ depot was formulated with Cur-M dispersed inside an outer shell of $\mathrm{CCH}$. It was expected that the $\mathrm{CCH}$ would act as an outer shell for the Cur-M, resulting in sustained Cur release from the microcapsules.

This is the first report of the development of an injectable, electrostatic, cross-linkable Cur-M-loaded $\mathrm{CCH}$ formulation that is suitable for intratumoral injection. As both the Cur-M-loaded CMC and Cur-M-loaded CHI formulations can be easily prepared as solution by mixing Cur-M with $\mathrm{CMC}$ and $\mathrm{CHI}$ solution, and then can form drug depot through minimally invasive chemotherapeutic administration, thus we believed that intratumoral injection of a CurM-loaded $\mathrm{CCH}$ formulation would synergistically inhibit tumor growth.

Accordingly, the objectives of this work were as follows: (1) to evaluate whether the prepared injectable, electrostatic, cross-linkable $\mathrm{CMC}$ and $\mathrm{CHI}$ formulation acts as a suitable Cur depot in vivo and (2) to determine whether the electrostatic, cross-linked $\mathrm{CCH}$ with Cur-M can produce synergistic tumor regression. Resolving these issues will help develop an efficient strategy for an injectable, electrostatic, crosslinkable Cur-M-loaded CCH formulation. Such a formulation holds the promise of achieving prolonged chemotherapeutic activity of safe and inexpensive Cur that is otherwise an unmet need with intratumoral injections.

\section{MATERIALS AND METHODS}

\section{Materials}

Cur (368.4 $\mathrm{g} \mathrm{mol}^{-1}$, Sigma, St Louis, MO, USA), poly(vinyl alcohol) (87-89\% hydrolyzed, molecular weight: 85000-124000 Da, Sigma), CMC sodium salt (CMC, low viscosity, Sigma, Steinheim, Germany), CHI (medium viscosity, 75-80\% deacetylated; Aldrich, Steinheim, Germany), FITC (Sigma), sodium azide (Sigma, MO, USA), IR-783 (Sigma), copper sulfate (Sigma), propargyl amine (TCI, Tokyo, Japan), N,N-dimethylformamide (JUNSEI, Tokyo, Japan) and Tween80 (Sigma) were purchased and used as received. PLGA (average molecular weight of $33 \mathrm{kDa}$ and lactic/glycolic acid ratio of 50:50) was used as received from Birmingham Polymers, Inc. (Birmingham, AL, UK). The sub- maxillary salivary gland cell line (epidermoid carcinoma A-253) was purchased from American Type Culture Collection (ATCC, Manassas, VA, USA). All other chemicals were of analytical grade and were used without further purification.

\section{Preparation of $\mathrm{CMC}$ and $\mathrm{CHI}$ formulations}

A $10 \%$ (w/v) CMC solution was prepared by dissolving CMC in $1 \times$ phosphatebuffered saline (PBS). The CHI solution was prepared by dissolving $400 \mathrm{mg}$ of $\mathrm{CHI}$ in $18 \mathrm{ml}$ of $0.1 \mathrm{M}$ acetic acid in deionized water (DW). Both CMC and $\mathrm{CHI}$ liquid solutions were clear and homogeneous. The CMC and CHI liquid solutions were separately loaded into each barrel of a dual-barrel syringe.

\section{Zeta potential measurements of CMC and $\mathrm{CHI}$ formulations}

Zeta potential of $0.36 \%(\mathrm{w} / \mathrm{v}) \mathrm{CMC}, 0.075 \%(\mathrm{w} / \mathrm{v}) \mathrm{CHI}$ and $\mathrm{CCH}$ was measured by dynamic light scattering (ELSZ-1000; Otsuka Electronics; Osaka, Japan) at $37^{\circ} \mathrm{C}$. $\mathrm{CCH}$ formulations with different ratios were prepared by varying the $\mathrm{CMC}: \mathrm{CHI}$ ratio from 100:0 to 0:100.

\section{Preparation of CMC-NIR and CHI-FITC for in vivo imaging}

To prepare CMC-NIR, we first synthesized NIR-783-N . NIR-783 $(0.3 \mathrm{~g}$, $0.45 \mathrm{mmol})$ and $\mathrm{N}, \mathrm{N}$-dimethylformamide (DMF) $(25 \mathrm{ml})$ were added to a flask. Sodium azide $(0.05 \mathrm{~g}, 0.9 \mathrm{mmol})$ was added to the NIR-783 solution at room temperature under nitrogen for $24 \mathrm{~h}$. The reaction mixture was poured into a mixture of ethyl acetate to precipitate crude NIR-783-N 3 . The NIR-783$\mathrm{N}_{3}$ was dissolved in ethanol and filtered. The filtrate was concentrated by evaporation and dried in a vacuum. To synthesize NIR-783- $\mathrm{NH}_{2}, \mathrm{NIR}-783-\mathrm{N}_{3}$ $(0.3 \mathrm{~g}, 0.45 \mathrm{mmol})$ was dissolved in DMF $(25 \mathrm{ml})$. Propargyl amine $(0.03 \mathrm{~g}$, $0.54 \mathrm{mmol})$, ascorbic acid $(0.16 \mathrm{~g}, 0.9 \mathrm{mmol})$ and $\mathrm{CuSO}_{4}(0.22 \mathrm{~g}, 0.9 \mathrm{mmol})$ were added into the NIR-783- $\mathrm{N}_{3}$ solution and stirred at room temperature overnight. The reaction mixture was poured into ethyl acetate to precipitate crude NIR-783-NH 2 . The NIR-783- $\mathrm{NH}_{2}$ was dissolved in ethanol and filtered. The filtrate was concentrated by evaporation and dried in a vacuum to give NIR-783- $\mathrm{NH}_{2}$.

As the next step, CMC $(100 \mathrm{mg})$ and $0.5 \mathrm{ml}$ methacrylic anhydride were dissolved in $50 \mathrm{ml} \mathrm{DW}$ and then stirred for $24 \mathrm{~h}$. Then, NIR-783-NH $(1 \mathrm{mg})$ was added to the reaction solution and the mixture stirred for another $24 \mathrm{~h}$. The unreacted IR-783- $\mathrm{NH}_{2}$ and methacrylic anhydride were removed by dialysis in DW for 3 days. The retentate was lyophilized using a freeze dryer at $-75^{\circ} \mathrm{C}$ to yield CMC-NIR.

For preparation of CHI-FITC, CHI $(100 \mathrm{mg})$ was dissolved in $50 \mathrm{ml} 0.1 \mathrm{M}$ acetic acid. FITC ( $1 \mathrm{mg}$ ) was added and the solution was stirred. After $24 \mathrm{~h}$, the unreacted FITC and acetic acid were removed by dialysis in DW for 3 days. The retentate was lyophilized using a freeze dryer at $-75^{\circ} \mathrm{C}$ to yield CHI-FITC. The detailed analysis was shown in Supplementary Figure S1.

\section{Preparation of Cur-M}

Cur-M was prepared using a monoaxial one-nozzle atomizer (Sono-Tek Crop, Milton, NY, USA). The typical preparation of Cur-M was achieved as follows: PLGA and Cur were dissolved in ethyl acetate and methanol, respectively. The concentrations of PLGA and Cur were $3 \%$ and $5 \% \mathrm{w} / \mathrm{v}$, respectively. The mixtures of PLGA and Cur were fed into the ultrasonic atomizer at flow rates of $4 \mathrm{ml} \mathrm{min}^{-1}$. Microdroplets were produced by atomizing the mixed solutions of PLGA and Cur for $\sim 5 \mathrm{~s}$ at a vibration frequency of $3 \mathrm{~W}$ per $60 \mathrm{kHz}$ and the microdroplets were then immediately collected in a $0.5 \% \mathrm{w} / \mathrm{v}$ poly(vinyl alcohol) solution for $2 \mathrm{~min}$. The distance between the atomizer head and the aqueous poly(vinyl alcohol) solution was $1 \mathrm{~cm}$, and the stirring speed of the poly(vinyl alcohol) solution was 1000 r.p.m. The resulting mixtures were gently stirred for $2 \mathrm{~h}$ to allow solidification of the microcapsules and were then filtered and washed with distilled water. The Cur-M was frozen at $-75^{\circ} \mathrm{C}$, followed by freeze-drying over 4 days. The morphology of Cur-M was confirmed using an optical microscope (Carl Zeiss Microimaging GmbH, Gottingen, Germany).

\section{Encapsulation efficiency of Cur-M}

The encapsulation efficiency of Cur was determined using acetonitrile and DW. Cur-M $(4 \mathrm{mg})$ was placed into a test tube and $0.6 \mathrm{ml}$ acetonitrile was added to 
dissolve the polymer portion of the microcapsules. Then, $1.4 \mathrm{ml}$ methanol and $2 \%$ acetic acid $(6: 1 \mathrm{v} / \mathrm{v})$ were added to dissolve the Cur. The resulting mixture was sonicated for $90 \mathrm{~min}$ at $25^{\circ} \mathrm{C}$ and centrifuged at 10000 r.p.m. for $5 \mathrm{~min}$.

The amount of Cur was determined using a high-performance liquid chromatography system (Agilent 1200 series, Waldbronn, Germany) with absorbance detection at $430 \mathrm{~nm}$. CAPCELL PACK C18 (RP18-415, $5 \mu \mathrm{m}$, $4.6 \times 250 \mathrm{~mm}$, SHISEIDO Co., Ltd, Tokyo, Japan) was used as a column. The mobile phase consisted of methanol, acetonitrile and $2 \%$ acetic acid $(6: 3: 1 \mathrm{v} / \mathrm{v})$, and the column was eluted at a flow rate of $1 \mathrm{ml} \mathrm{min}^{-1}$. Three independent release experiments were performed for each Cur-M. The encapsulation efficiency $(E)$ was defined as follows:

$$
E=[(\text { amount of encapsulated Cur }) /(\text { total amount of Cur added })] \times 100
$$

\section{Preparation of Cur-M-loaded CCH}

Cur-M (60 mg) was suspended in $1 \mathrm{ml}$ of solution (5\% D-mannitol, $2 \%$ CMC and $0.1 \%$ Tween 80 ) and $4 \mathrm{ml}$ of PBS solution (Cur-M alone formulation). For in vitro Cur release experiments, the prepared Cur-M solution was added to the $\mathrm{CCH}$ in a $5 \mathrm{ml}$ vial and gently mixed. For in vivo antitumor experiments, an equal amount of Cur-M was individually mixed in the $\mathrm{CHI}$ and $\mathrm{CMC}$ solutions. Cur-M-loaded CHI solution and Cur-M-loaded CMC solution were separately charged in each barrel of dual-barrel syringe (Cur-M-loaded CCH formulation).

To assess the Cur-M dispersed inside an outer shell of CCH, $200 \mu \mathrm{l}$ of CurM-loaded CHI solution and Cur-M-loaded CMC solution were injected into the subcutaneous tissue of mice using 21-G syringes. The Cur-M-loaded CCH was removed from the mouse at days 1 and 20, and was observed by scanning electron microscopy (SEM), as described in the next section.

\section{SEM observation of injectable formulations}

SEM was performed with a JSM-6700F (JEOL, Tokyo, Japan), to examine the morphology of the in vitro and in vivo prepared Cur-M, CCH and Cur-Mloaded $\mathrm{CCH}$. The Cur-M, $\mathrm{CCH}$ and Cur-M-loaded $\mathrm{CCH}$ were mounted on a metal stub that was precooled in liquid nitrogen. The metal stub was quickly immersed in a liquid nitrogen bath to minimize alteration of the Cur-M, $\mathrm{CCH}$ and Cur-M-loaded CCH. The stub was freeze-dried at $-75^{\circ} \mathrm{C}$. The dried Cur$\mathrm{M}, \mathrm{CCH}$ and Cur-M-loaded $\mathrm{CCH}$ on the metal stub was coated with a thin layer of gold using a plasma sputtering apparatus (Cressington 108 Auto Sputter Coater, Ted Pella, Inc., Redding, CA, USA) under an argon atmosphere.

\section{Rheological measurements}

Rheological properties were measured using a modular compact rheometer (MCR 102, Anton Paar, Graz, Austria) with a temperature-controlled bottom platen. The parallel plate diameter used was $25 \mathrm{~mm}$ and the distance between the plates was $0.3 \mathrm{~mm}$ at room temperature and $37^{\circ} \mathrm{C}$. A frequency sweep was measured between 0.1 and $10 \mathrm{~Hz}$ of the oscillating frequency at a strain of $2 \%$. The viscosity was measured at a constant frequency of $1 \mathrm{~Hz}$. All rheological properties were analyzed with the instrument software (Rheoplus/32, version V3.21, Anton Paar)

\section{In vivo imaging of injectable formulations}

The CMC-NIR and CHI-FITC solutions were injected into the subcutaneous tissue of mice. CMC-NIR and CHI-FITC solution $(200 \mu \mathrm{l})$, separately charged in each barrel of a dual-barrel syringe, were injected into subcutaneous tissue using 21-G syringes. Cur-M and Cur-M-loaded CCH (200 $\mu \mathrm{l})$ were intratumorally injected using 21-G syringes. For fluorescence excitation $460 \mathrm{~nm}$ light was used and for emission light was filtered with a $500-525 \mathrm{~nm}$ band-pass filter. At each time point, fluorescence images were acquired using an imaging instrument (FOBI, NeoScience, Suwon, Korea). NIR images were taken with an exposure time of $100 \mathrm{~s}$ and gain of one, using a dichroic cube filter $\left(\mathrm{MgF}_{2}\right.$, fused silica filter).

\section{In vitro release}

Cur-M alone (Cur $4 \mathrm{mg}$ ) and a mixture of Cur-M (Cur $4 \mathrm{mg}$ ) were separately transferred to fresh $5 \mathrm{ml}$ vials, suspended in $4 \mathrm{ml}$ of $\mathrm{PBS}$ ( $\mathrm{pH}$ 7.4) and shaken in an incubator at 100 r.p.m. and $37^{\circ} \mathrm{C}$. At predetermined time intervals, $500 \mu \mathrm{l}$ of solution was removed from each vial and replaced with $500 \mu$ l of fresh PBS containing $0.1 \%$ Tween 80 solution at $37^{\circ} \mathrm{C}$. The amount of released Cur was determined using high-performance liquid chromatography, as described in the previous section. Four independent release experiments were performed for each experiment. The amount of Cur cumulatively released in vitro was calculated by comparison with standard calibration curves prepared with known concentrations of Cur.

\section{In vitro antitumor activity}

A-252 tumor cells $\left(2 \times 10^{4}\right.$ cells per well) were seeded in the lower chambers of a 24-well Transwell plate (SPL Life Science, $0.4 \mu \mathrm{m}$ pore size, Pocheon, Gyeonggi, South Korea) and incubated for 1 day at $37^{\circ} \mathrm{C}$ in a humidified incubator containing 5\% $\mathrm{CO}_{2}$. One hundred and fifty microliters of PBS, microcapsule alone, $\mathrm{CCH}$ alone, free Cur $(0.8 \mathrm{mg}$ Cur per well), Cur-M (0.8 mg Cur per well) or Cur-M/CCH $(0.8 \mathrm{mg}$ Cur per well) were added directly to the upper chambers of the Transwell plate and the incubation continued for $1 \mathrm{~h}$. Each upper chamber was then placed above a lower chamber containing A-253 cells $\left(2 \times 10^{4}\right.$ cells per well).

For all formulations, the initial medium was changed after $12 \mathrm{~h}$. After 24, 48 and $72 \mathrm{~h}$, the in vitro cytotoxicity of all formulations toward the A-253 cells was compared using an MTT (3-(4,5-dimethylthiazol-2-yl)-2,5-diphenyltetrazolium bromide; Sigma-Aldrich Co., St Louis, MO, USA) assay. In brief, $100 \mu \mathrm{l}$ of MTT solution ( $50 \mu \mathrm{g} \mathrm{ml}^{-1}$ in PBS) was added to each well plate and the plates were incubated at $37^{\circ} \mathrm{C}$ for $4 \mathrm{~h}$. The resulting violet formazan precipitate was solubilized by the addition of $500 \mu \mathrm{l}$ dimethyl sulfoxide and shaking for $30 \mathrm{~min}$. The solutions were placed in 96-well plates and read using a microplate reader (E-max, Molecular Devices, Sunnyvale, CA, USA). The optical density of each well was measured at a wavelength of $590 \mathrm{~nm}$. All experiments were performed at least four times and the results are presented as mean \pm s.d.

\section{In vivo antitumor activity}

The protocols of this study were approved by the Institutional Animal Experiment Committee of the School of Medicine of Ajou University. Animal experiments were carried out in accordance with approved guidelines.

The tumor model was established by subcutaneous inoculation of A-253 $\left(1 \times 10^{7}\right.$ cells, in a $0.2 \mathrm{ml}$ suspension $)$ in the abdomen of each BALB/c nude mouse (5-6 weeks, 16-17 g). The time when the solid tumor volume reached $110 \pm 30 \mathrm{~mm}^{3}$ was defined as day 0 . The animals were assigned to five experimental groups, that is, (1) normal saline, (2) single injection of free Cur $\left(4 \mathrm{mg} \mathrm{ml}^{-1}\right)$, (3) repeated injections of free Cur $\left(4 \mathrm{mg} \mathrm{ml}^{-1}\right.$ per injection), (4) Cur-M alone (4 mg ml${ }^{-1}$ of Cur) and (5) Cur-M-loaded CCH (4 mg ml${ }^{-1}$ of Cur). On day 0, $200 \mu \mathrm{l}$ of each solution was injected directly into the tumor using a 21-gauge needle and disposable syringe $(1 \mathrm{ml})$. Animals in the repeated injections group (group 3) were injected again on days 4, 9 and 14. Antitumor activity was assessed by measuring tumor diameters in two dimensions with Vernier calipers at predefined days. The tumor volume $(V)$ was calculated according to the following formula: $V=\left[\right.$ length $\left.\times(\text { width })^{2}\right] / 2$.

For pharmacokinetics, experimental animals were killed on days 1,10 and 20 by cervical dislocation and the organs (tumor, intestine, stomach, lung, kidney, liver, spleen and heart) were collected immediately. Each organ was homogenized in distilled water using a T10 basic ULTRA-TURRAX Homogenizer (IKA, Werke GMBH, Königswinter, Germany) at 6000 r.p.m. for $15 \mathrm{~min}$, and then incubated at $37^{\circ} \mathrm{C}$ for $15 \mathrm{~min}$. Each sample was mixed with an equal volume of $40 \%$ (w/v) $\mathrm{ZnSO}_{4}$ and mobile phase (as described in the previous section), and incubated again at $37^{\circ} \mathrm{C}$ for $15 \mathrm{~min}$. The amount of Cur in the supernatant, obtained after centrifugation at 2000 r.p.m. for $10 \mathrm{~min}$, was determined using high-performance liquid chromatography as described in the previous section. Three independent experiments were performed for each organ.

\section{Histological analysis}

On days 1,10 and 20 after initial injection, mice were killed and the tumors were excised from the subcutaneous abdomen. The tissues were immediately fixed with $10 \%$ formalin and embedded in paraffin. The embedded specimens were sectioned $(4 \mu \mathrm{m})$ along the longitudinal axis of the tumor and incubated 
at $70{ }^{\circ} \mathrm{C}$ for $2 \mathrm{~h}$ to remove excess paraffin. The slides were further deparaffinized with xylene twice and hydrated using 100, 95, 70 and $60 \%$ ethyl alcohol sequentially. For hematoxylin and eosin staining, samples were washed in running tap water and stained with hematoxylin and eosin for 3 min. Thereafter, stained slides were mounted with mounting medium (Muto Pure Chemicals, Tokyo, Japan).

Apoptotic cells were identified using a Terminal deoxynucleotidyl transferase dUTP Nick-End Labeling (TUNEL) assay kit (In Situ Cell Death Detection Kit, Roche, Mannheim, Germany), following the manufacturer's protocol. Briefly, the slides were deparaffinized at $70{ }^{\circ} \mathrm{C}$, hydrated and washed three times with PBS-T (0.1\% Tween20 in PBS) for $10 \mathrm{~min}$ per wash. The tissues were incubated with $1 \mathrm{mg} \mathrm{ml}^{-1}$ proteinase $\mathrm{K}$ (Invitrogen, Carlsbad, CA, USA) in TE buffer at $37^{\circ} \mathrm{C}$ for $20 \mathrm{~min}$ and then washed with PBS-T. The tissues were incubated with $0.1 \%$ Triton X-100 at $4{ }^{\circ} \mathrm{C}$ for 5 min and then treated with a mixture of labeling solution and enzyme solution $(9: 1)$ at $37^{\circ} \mathrm{C}$ for $60 \mathrm{~min}$. The slides were counterstained with 4',6-diamidino-2-phenylindole and then mounted with fluorescent mounting medium (DAKO, Carpinteria, CA, USA). Fluorescent images were obtained using an Axio Imager A1 (Carl Zeiss Microimaging $\mathrm{GmbH}$ ) and analyzed with Axiovision Rel. 4.8 software (Carl Zeiss Microimaging $\mathrm{GmbH}$ ).

\section{Statistical analysis}

Tumor sizes were evaluated in experimental groups with $n=3$ for all data points. Organ Cur contents were obtained from independent experiments, in which each of the five treatment conditions were tested in triplicate. Results of the TUNEL assay were obtained in triplicate from independent experiments. All data are shown as means \pm s.d. The data from each experiment were examined using one-way analysis of variance with Bonferroni's multiple comparisons. Statistical analyses were performed with SPSS 12.0 software (IBM Corporation, Armonk, NY, USA).

\section{RESULTS}

Characterization of injectable CMC and CHI formulations

Based on previous work regarding hydrogel formation via electrostatic interactions, ${ }^{3}$ as shown in Figure 1, we chose the anionic CMC and cationic $\mathrm{CHI}$ solutions to form an instantaneous drug depot after intratumoral injection. Each $\mathrm{CMC}$ and $\mathrm{CHI}$ solution flowed when tilted at room temperature and at $37^{\circ} \mathrm{C}$. The $\mathrm{CMC}$ and $\mathrm{CHI}$ were distinct solutions prior to mixing (Figures 2a and b), but readily formed the $\mathrm{CCH}$ hydrogel after mixing (Figure 2c).

Thus, to understand the electrostatic interaction between the anionic $\mathrm{CMC}$ and cationic $\mathrm{CHI}$, zeta potential was measured for solutions containing varying ratios of $\mathrm{CMC}$ and $\mathrm{CHI}$ at $37^{\circ} \mathrm{C}$ (Figure 3). The zeta potentials of $\mathrm{CMC}$ and $\mathrm{CHI}$ solutions were -21 and $+42 \mathrm{mV}$, respectively. The zeta potential increased as the amount of $\mathrm{CHI}$ increased.

The viscosity change after mixing $\mathrm{CMC}$ and $\mathrm{CHI}$ solutions was measured to investigate the electrostatic interaction between the anionic $\mathrm{CMC}$ and cationic $\mathrm{CHI}$. The $\mathrm{CMC}$ and $\mathrm{CHI}$ solutions were clear with viscosities of $3.5 \mathrm{~Pa} \cdot \mathrm{s}$. and $8.2 \mathrm{~Pa} \cdot \mathrm{s}$. at $37^{\circ} \mathrm{C}$, respectively. A 50:50 (w/w) mixture of $\mathrm{CHI}$ and $\mathrm{CMC}$ (that is, $\mathrm{CCH}$ ) became a hydrogel at $37^{\circ} \mathrm{C}$, exhibiting a viscosity of $135 \mathrm{~Pa} \cdot \mathrm{s}$. The viscosity changes for $\mathrm{CCH}$ hydrogels of varying proportions produced a parabolic curve with a maximum viscosity at equal proportions of $\mathrm{CHI}$ and $\mathrm{CMC}$ (Figure 3). These results indicate that $\mathrm{CMC}$ and $\mathrm{CHI}$ solutions exist as a liquid before mixing, and form a $\mathrm{CCH}$ hydrogel as a drug depot via electrostatic interactions between the anionic CMC and cationic $\mathrm{CHI}$ after mixing. The morphology of the electrostatic, cross-linked $\mathrm{CCH}$ hydrogel revealed a network of interconnected three-dimensional structures (Supplementary Figure S2).

\section{Preparation of Cur-M}

Cur-M was prepared with a mono axial nozzle ultra-sonic atomizer, as previously reported (Figure 2d). ${ }^{19}$ The yield of preparation was $75 \pm 5 \%$. As shown in optical, fluorescence and SEM images, Cur-M microcapsules were spherical with a smooth surface and yellowish hue due to the original color of Cur (Figures $2 \mathrm{e}-\mathrm{g}$ ). The mean particle size of Cur-M was $46 \mu \mathrm{m}$. The Cur encapsulation efficiency was $76 \pm 10 \%$.

\section{Preparation of Cur injectable formulations}

Cur-M/CMC and Cur-M/CHI solution formulations can be prepared easily by mixing Cur-M with $\mathrm{CMC}$ and $\mathrm{CHI}$ solution, respectively. Cur-M/CMC and Cur-M/CHI solutions were translucent, yellowish suspensions (Figures $2 \mathrm{~h}$ and i). Mixing Cur-M-loaded CMC solution

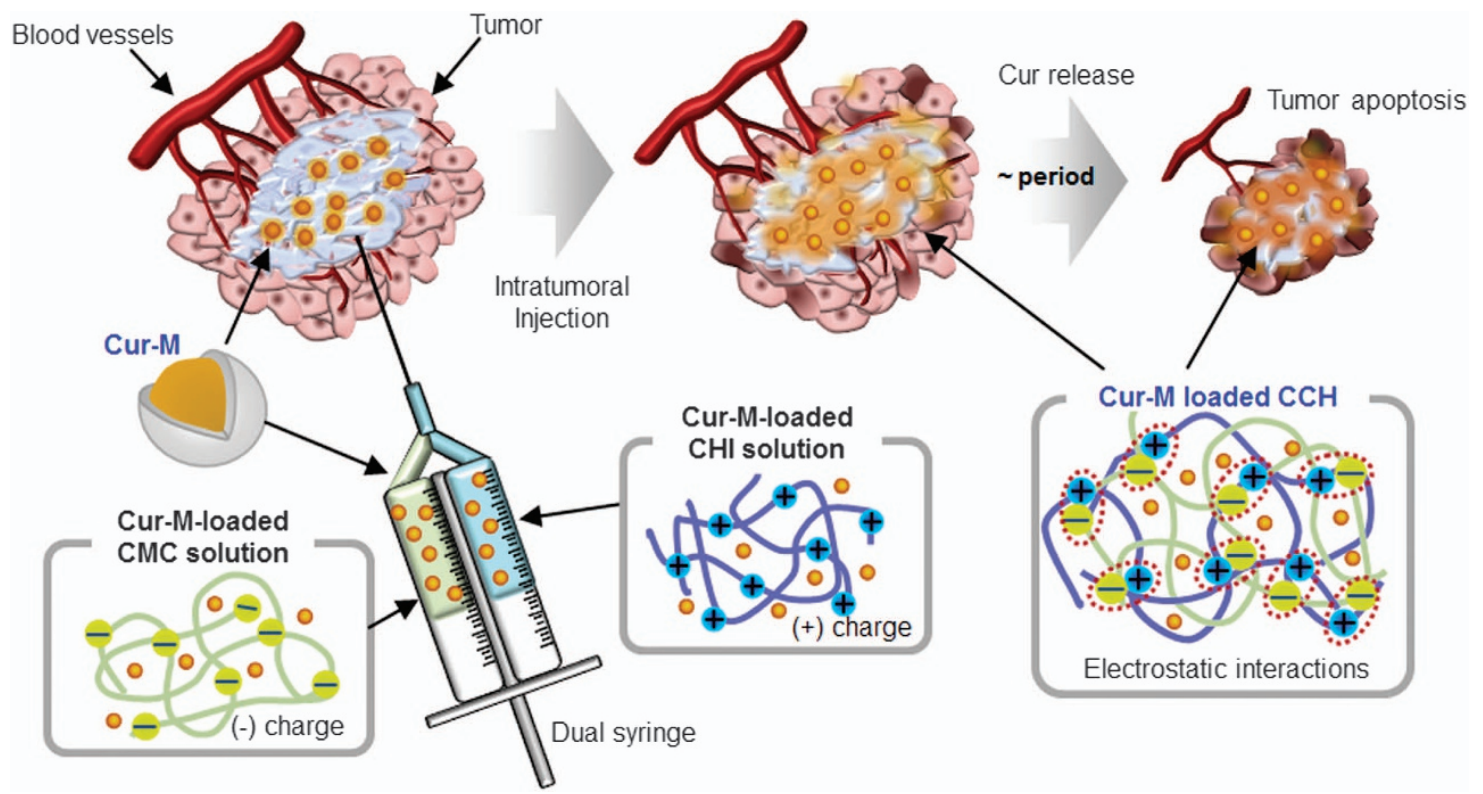

Figure 1 Schematic representation of synergistic tumor suppression via intratumoral injections of Cur-M loaded $\mathrm{CCH}$ drug depots and controlled release of Cur. 


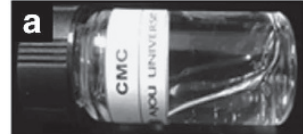

CMC solution
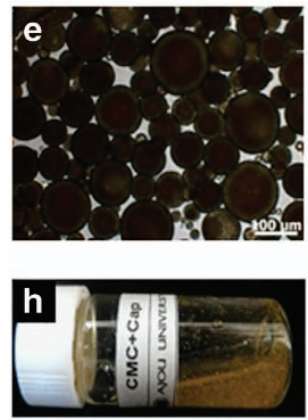

Cur-M-loaded $\mathrm{CMC}$ solution

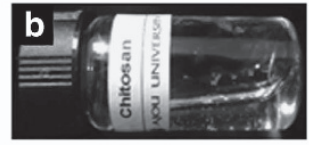

$\mathrm{CHI}$ solution
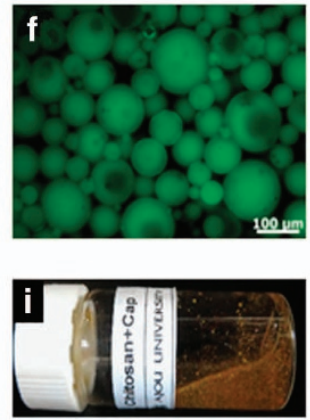

Cur-M-loaded $\mathrm{CH}$ solution

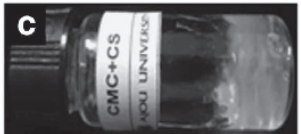

$\mathrm{CCH}$
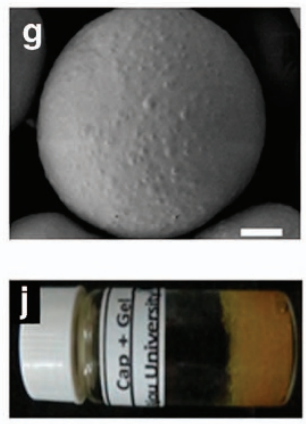

Cur-M-loaded $\mathrm{CCH}$

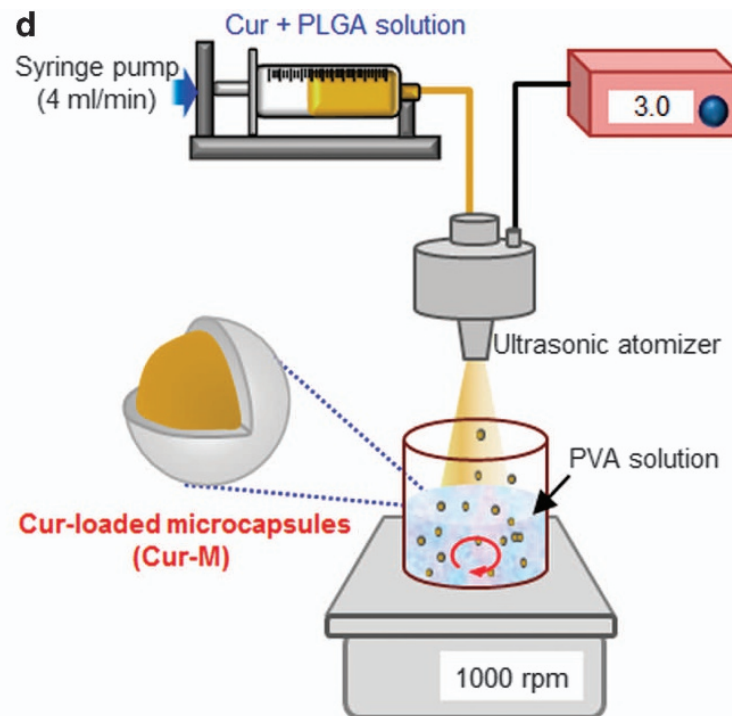

Figure 2 Pictures of (a) $\mathrm{CMC}$ solution, (b) $\mathrm{CHI}$ solution and (c) $\mathrm{CCH}$; (d) schematic representation of the preparation of Cur-M using a mono-axial ultrasonic atomizer; (e) optical microscopy (scale bar: $100 \mu \mathrm{m}$ ), (f) fluorescence microscopy (scale bar: $100 \mu \mathrm{m}$ ) and (g) scanning electron microscopy of Cur-M (SEM: $\times 800$ magnification, scale bar: $10 \mu \mathrm{m}$ ); (h) Cur-M-loaded CMC solution, (i) Cur-M-loaded CHI solution and (j) Cur-M-loaded CCH.

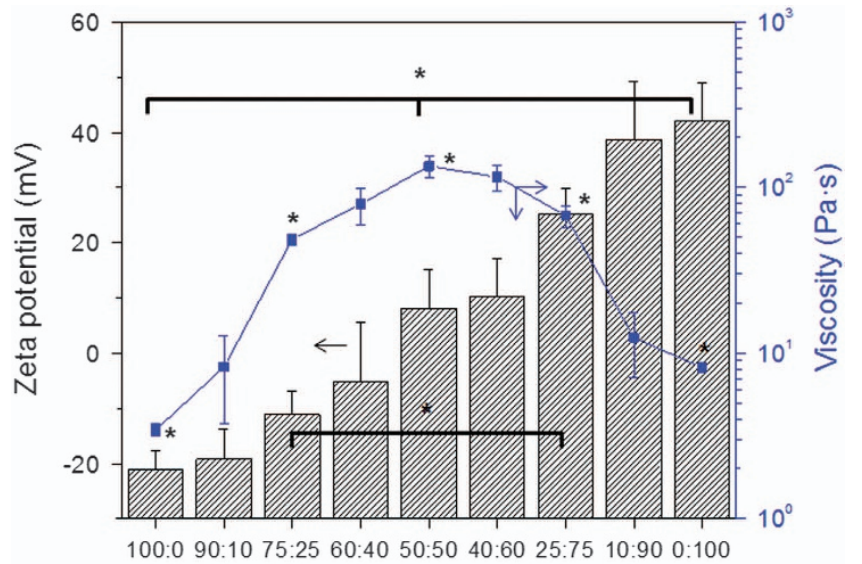

$\mathrm{CMC}: \mathrm{CHI}$ ratio

Figure 3 Zeta potential and viscosity versus ratios of $\mathrm{CMC}$ and $\mathrm{CH}$ solution $\left({ }^{*} P<0.001\right)$ (black and blue arrow indicate zeta potential and viscosity, respectively).

with Cur-M-loaded CHI solution readily formed Cur-M-loaded $\mathrm{CCH}$ hydrogel (Figure 2j).

The gelation and mechanical properties of the injectable formulations were further analyzed by rheology (Figure 4). In the frequency sweep test, the storage modulus values show the mechanical stability over a change of frequency from 0.1 to $10 \mathrm{~Hz}$ at $37^{\circ} \mathrm{C}$. $\mathrm{CCH}$ exhibited higher storage modulus values than those of the individual CMC and $\mathrm{CHI}$ solutions at all frequency ranges tested $(0.1$ to $10 \mathrm{~Hz}$ ) (Figure $4 \mathrm{a}$ ), indicating a higher mechanical stiffness of $\mathrm{CCH}$.

The storage $\left(G^{\prime}\right)$ and loss $\left(G^{\prime \prime}\right)$ modulus values of CMC and CHI solution without or with Cur-M and $\mathrm{CCH}$ or Cur-M-loaded $\mathrm{CCH}$ hydrogels were measured at $37^{\circ} \mathrm{C}$ at a frequency of $1 \mathrm{~Hz}$ (Figures $4 \mathrm{~b}$ and c). The storage values of $\mathrm{CMC}$ and $\mathrm{CHI}$ solution without or with Cur-M were lower than those of Cur-M-loaded $\mathrm{CCH}$ hydrogels at $37^{\circ}$ C. CMC and CHI solutions without or with Cur-M had similar loss modulus and storage modulus values at $37^{\circ} \mathrm{C}$, but $\mathrm{CCH}$ or Cur-M- loaded $\mathrm{CCH}$ hydrogel had lower loss modulus values, indicating the elastic, gel-like characteristics of $\mathrm{CCH}$.

In addition, $\mathrm{CCH}$ or Cur-M-loaded $\mathrm{CCH}$ exhibited greater viscosity at $37^{\circ} \mathrm{C}$ than that of $\mathrm{CMC}$ and $\mathrm{CHI}$ solution without or with Cur-M (Figure $4 \mathrm{~d}$ ). $\mathrm{CCH}$ or Cur-M-loaded $\mathrm{CCH}$ showed a decreased phase angle (tan delta), compared with that of $\mathrm{CMC}$ and $\mathrm{CHI}$ solution. The phase angle (tan delta) values of $\mathrm{CCH}$ or Cur-M-loaded $\mathrm{CCH}$ were about 0.3 (Figure 4e). This indicates that $\mathrm{CCH}$ or Cur-M-loaded $\mathrm{CCH}$ exhibits gel-like features, because a value of tan delta $<1$ describes a predominantly elastic gel behavior. ${ }^{25}$

Collectively, these results indicate that the $\mathrm{CCH}$ or Cur-M-loaded $\mathrm{CCH}$ had significantly stiffer, hydrogel-like features than $\mathrm{CMC}$ and CHI solutions did at $37^{\circ} \mathrm{C}$.

\section{In vivo formation of Cur drug depot}

To examine the ability of $\mathrm{CMC}$ and $\mathrm{CHI}$ solutions to form an electrostatic, cross-linked Cur-M-loaded CCH depot, Cur-M-loaded CMC and Cur-M-loaded CHI solutions were separately loaded into each barrel of a dual-barrel syringe (Figure $5 \mathrm{a}$ ). The solutions were then co-injected into mice (Figure 5b). The formulation solution gelled in the mice almost simultaneously with subcutaneous injection and the gelled $\mathrm{CCH}$ depot was maintained for at least 20 days.

The resulting electrostatic, cross-linked $\mathrm{CCH}$ and Cur-M-loaded $\mathrm{CCH}$ depots were allowed to develop in vivo and were biopsied 20 days after implantation (Figures $5 \mathrm{c}-\mathrm{f}$ ). The electrostatic, crosslinked $\mathrm{CCH}$ maintained structure at the injection site for the full experimental observation period, although the size decreased at day 20 (Figures $5 \mathrm{e}$ and $\mathrm{f}$ ).

SEM images of the in vivo electrostatic, cross-linked Cur-M-loaded $\mathrm{CCH}$ depot revealed that the Cur-M was interspersed in the $\mathrm{CCH}$ hydrogel (Figures $5 \mathrm{~g}$ and $\mathrm{h}$ ), indicating that the Cur-M was inside the $\mathrm{CCH}$ hydrogel.

Collectively, these results indicate that the Cur-M-loaded $\mathrm{CCH}$ formulations had Cur-M dispersed inside the $\mathrm{CCH}$; thus, the $\mathrm{CCH}$ hydrogel acted as an additional outer shell for the Cur-M. These results also indicate that the electrostatic, cross-linked, Cur-M-loaded 
a

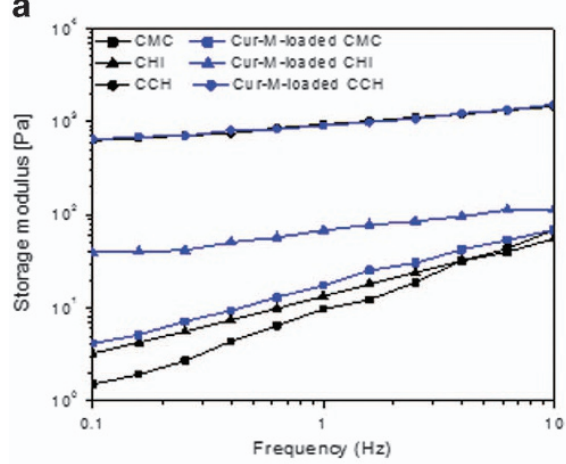

b

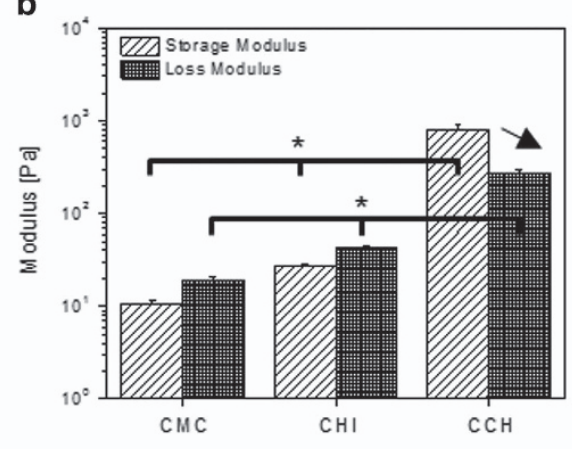

C

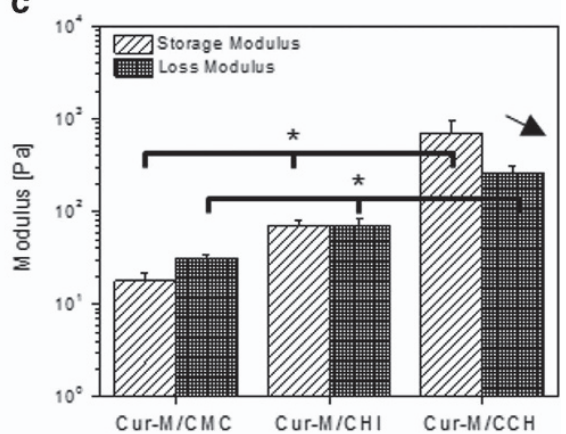

d

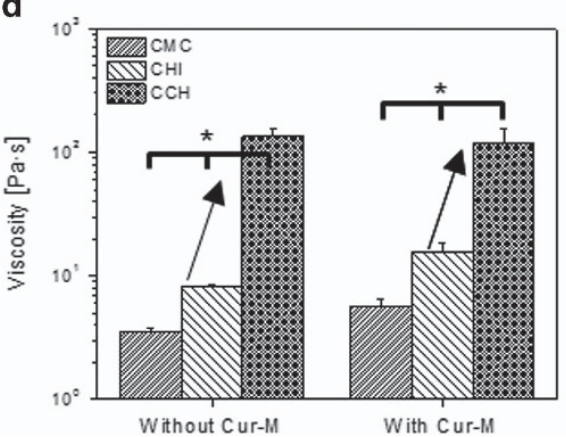

e

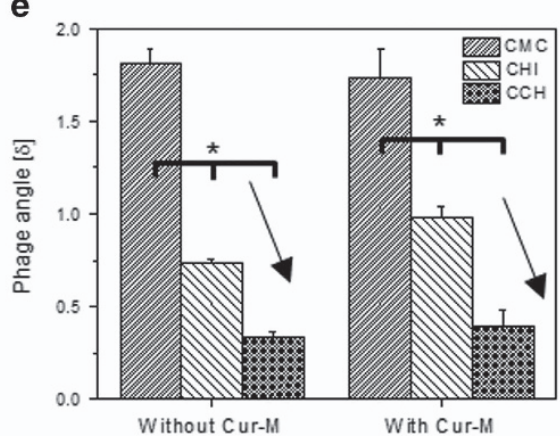

Figure 4 Rheological characterization of (a) storage modulus versus frequency sweep test of CMC only, $\mathrm{CHI}$ only, CCH only, Cur-M-loaded CMC, Cur-M-loaded $\mathrm{CHI}$ and $\mathrm{Cur}-\mathrm{M}$-loaded $\mathrm{CCH}$; (b) storage and loss modulus of $\mathrm{CMC}$ only, $\mathrm{CHI}$ only and $\mathrm{CCH}$ only; (c) storage and loss modulus of Cur-M-loaded $\mathrm{CMC}$, Cur-Mloaded $\mathrm{CHI}$ and Cur-M-loaded $\mathrm{CCH}$; (d) viscosity of $\mathrm{CMC}, \mathrm{CHI}$ and $\mathrm{CCH}$ with and without $\mathrm{Cur}-\mathrm{M}$; (e) phase angle of $\mathrm{CMC}, \mathrm{CHI}$ and $\mathrm{CCH}$ with and without Cur-M $\left({ }^{*} P<0.001\right)$.

$\mathrm{CCH}$ depot can maintain sufficient structural integrity to act as a drug depot in vivo and suggest that Cur is released at the injection site.

\section{In vivo persistence of injectable materials}

To examine in vivo $\mathrm{CHI}$ and $\mathrm{CMC}$ persistence using real-time imaging, CHI-FITC and CMC-NIR were prepared and injected separately into subcutaneous tissue of mouse to examine in vivo $\mathrm{CHI}$ and CMC persistence (Figures 6a and b). Fluorescent and NIR images were observed $30 \mathrm{~min}$ after injecting CHI-FITC and CMCNIR, respectively (Figures $6 \mathrm{a}$ and $\mathrm{b}$ ), due to distinct $\mathrm{CHI}$ and $\mathrm{CMC}$ hydrogel formation. However, negligible fluorescent and NIR images were observed at days 3 and 2, respectively (Figures 6a and b), and no signal was observed after 4 and 3 days, respectively, indicating a rapid clearance of CHI-FITC and CMC-NIR from subcutaneous tissue.

To examine in vivo electrostatic, cross-linked $\mathrm{CCH}$ persistence using real-time imaging, CHI-FITC and CMC-NIR were separately filled into each barrel of a dual-barrel syringe and were co-injected into subcutaneous tissue of mouse (Figure 6d). The injectable CHI-FITC and CMC-NIR formulation formed electrostatic cross-linked $\mathrm{CCH}$ depots. Fluorescent and NIR signals of $\mathrm{CCH}$ were observed in the subcutaneous tissue shortly after injection, indicating the formation of in vivo electrostatic, cross-linked $\mathrm{CCH}$ depot. The signals could be detected for at least 21 days after injection. Therefore, we confirmed that $\mathrm{CMC}$ and $\mathrm{CHI}$ solutions successfully formed in vivo electrostatic, cross-linked CCH depots. Furthermore, the electrostatic, cross-linked $\mathrm{CCH}$ depot persisted in vivo for an extended period, compared with the individual CMC and $\mathrm{CHI}$ solutions.

\section{In vitro Cur release from Cur injectable formulations}

The in vitro Cur-release behavior from Cur-M alone and Cur-M dispersed inside $\mathrm{CCH}$ was examined at $37^{\circ} \mathrm{C}$ for 22 days
(Supplementary Figure S3). The cumulative amount of Cur released from the Cur-M alone was $30 \%$ at day 5, due to an initial burst, and then $48 \%$ release of Cur by day 22. For Cur-M dispersed inside $\mathrm{CCH}$, the Cur release was $12 \%$ at day 5 , followed by a slower release, totaling $33 \%$ by day 22 . This retardation was due to the $\mathrm{CCH}$ hydrogel acting as an outer, protective shell for the Cur-M. Importantly, the initial burst of Cur release was small and the release pattern was linear.

\section{Anti-proliferative effects of Cur injectable formulations}

The anti-proliferative activities of PBS, microcapsule alone, $\mathrm{CCH}$ alone, free Cur, Cur-M and Cur-M-loaded $\mathrm{CCH}$ were assessed with A-253 tumor cells (Figure 7a). Cells treated with PBS showed a timedependent increase in numbers. After adding PBS, microcapsule alone or $\mathrm{CCH}$ alone, cells proliferated and their numbers increased by $40-50 \%$ at day 2 and $60-70 \%$ at day 3, compared with cell numbers at day $1\left({ }^{*} P<0.001\right)$.

The cell viability with free Cur was $<22 \%$ at day 1 , compared with that of PBS-treated cells, indicating that free Cur had anti-proliferative effects in A-253 tumor cells. However, the cells recovered and proliferated thereafter $\left({ }^{*} P<0.05\right)$.

For Cur-M, cell viability was below $34 \%$ at day 1 and the viability was maintained at $\sim 30 \%$ in comparison with that reported for the control cells. Compared with that of free Cur, Cur-M effectively inhibited cell proliferation at day $3\left({ }^{*} P<0.001\right)$. This result indicates that the Cur released from Cur-M can inhibit cell proliferation.

The cell viability during exposure to Cur-M-loaded $\mathrm{CCH}$ was $\sim 40 \%$ at day 1 . Cell viability was slightly higher than that observed with free Cur or Cur-M, because the $\mathrm{CCH}$ hydrogel slowed the release of Cur. However, cell viability gradually decreased to $\sim 19 \%$ at day 2 and $8 \%$ at day 3 . These results indicate that Cur-M-loaded $\mathrm{CCH}$ can 

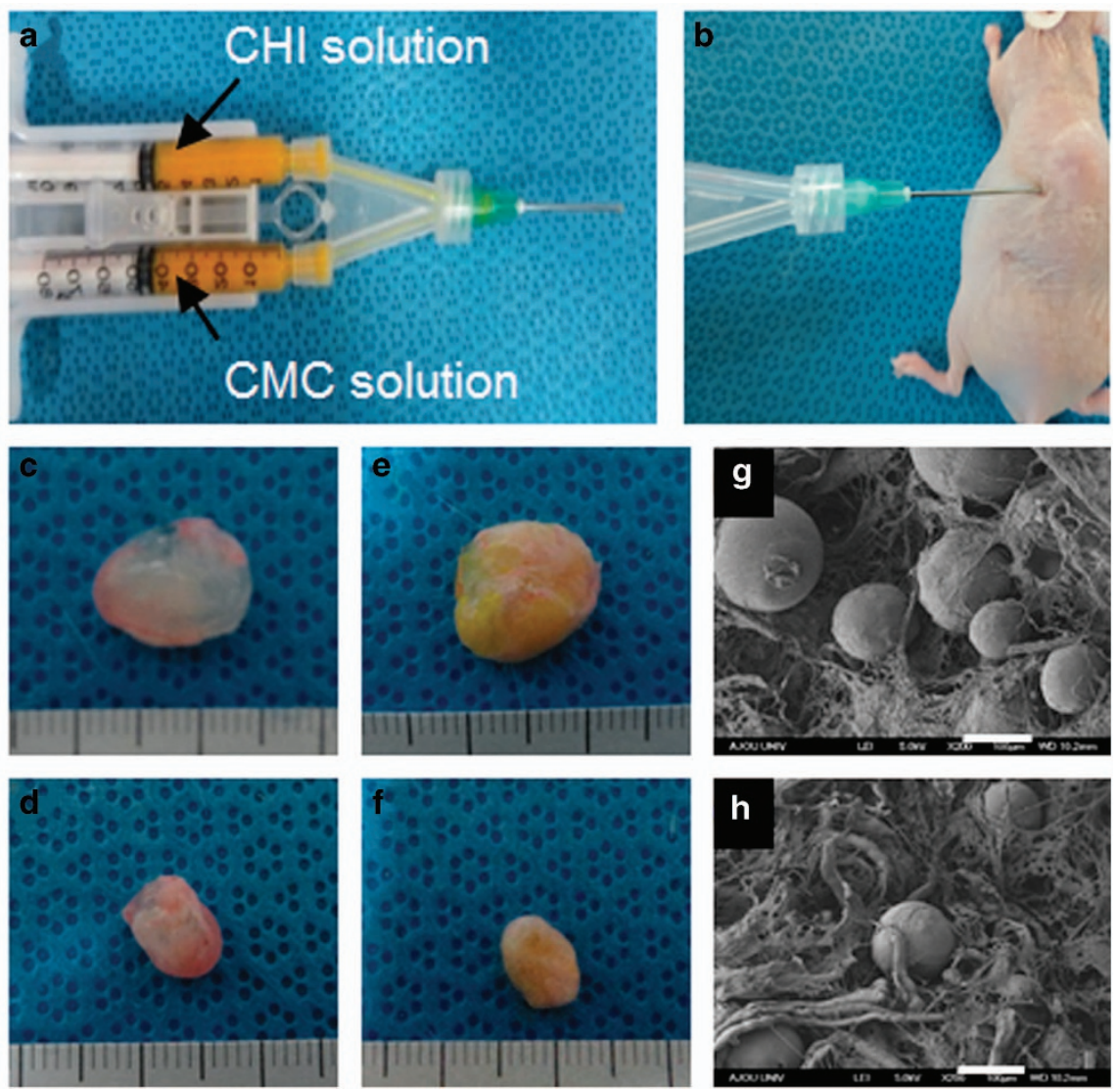

Figure 5 Pictures of (a) dual-injection formulations; (b) subcutaneous injection of Cur-M loaded CCH into mice; (c, d) the removed CCH at (c) day 1 and (d) 20; (e, f) the removed drug depot of Cur-M loaded CCH at (e) day 1 and (f) 20; (g, h) scanning electron microscopy (SEM: $\times 200$ magnification, scale bar: $100 \mu \mathrm{m}$ ) images for the removed drug depot of Cur-M loaded $\mathrm{CCH}$ at (g) day 1 and (h) 20.

produce nearly complete anti-proliferative effects due to the sustained release of Cur from the $\mathrm{CCH}$ hydrogel that acts as an outer shell for the Cur-M.

Optical and fluorescent images of A-253 cells were obtained after exposure to PBS, free Cur, Cur-M and Cur-M-loaded CCH (Figure 7b and Supplementary Figure S4). Blue and green fluorescence is indicative of 4',6-diamidino-2-phenylindole -stained nuclei and Cur, respectively. The merged images show sky blue fluorescence due to the overlay of the blue and green fluorescent images.

The control cells showed an increase in population in the optical and blue fluorescent images from 1 to 3 days, with no green fluorescence. The cells treated with free Cur showed blue, green and sky blue fluorescence at day 1 . Blue fluorescence was maintained, but green and sky blue fluorescence disappeared at days 2 and 3.

The cells treated with Cur-M showed a decrease in the optical and blue fluorescence, but green and sky blue fluorescence was maintained for 3 days. These results strongly suggest that the Cur released from the Cur-M induced A-253 cell death.

In the cells treated with Cur-M-loaded $\mathrm{CCH}$, the blue fluorescence decreased with culture time. Slight green fluorescence was observed at day 1 , but it increased at days 2 and 3, suggesting a sustained release of Cur from the $\mathrm{CCH}$ hydrogel. Sky blue fluorescence was observed in the nuclei of A-253 cells in the merged images, which decreased further with incubation time. These results suggest that the sustained release of Cur from the $\mathrm{CCH}$ hydrogel induced almost complete death of the A-253 cells.

\section{In vivo Cur release from Cur injectable formulations}

To examine the in vivo persistence of Cur release using real-time imaging, free Cur, Cur-M and Cur-M-loaded $\mathrm{CCH}$ were injected into the subcutaneous tissue of mouse (Figure 8). Injection of free Cur, Cur-M and Cur-M-loaded $\mathrm{CCH}$ produced green fluorescent signals at the initial $30 \mathrm{~min}$ time point. The fluorescence of injected free Cur dropped significantly by $10 \mathrm{~h}$ and was completely lost by day 4 , due to rapid systemic elimination of Cur from subcutaneous tissue (Figure 8a).

For the Cur-M-injected mouse, fluorescence of Cur-M was observed in subcutaneous tissue after injection, indicating the formation of the in vivo Cur-M depot. The fluorescent signals persisted for at least 10 days after injection, but disappeared by day 12 (Figure $8 b$ ).

Meanwhile, in the mouse that received an injection of Cur-Mloaded $\mathrm{CCH}$, fluorescent signals persisted for 20 days after injection (Figure 8c); negligible fluorescence was observed by day 22. Thus, the Cur in the formulation of Cur-M-loaded $\mathrm{CCH}$ was maintained in vivo for at least 20 days. This result indicates that in vivo Cur can persist for an extended period because of the $\mathrm{CCH}$ hydrogel acting as an outer shell for the Cur-M.

\section{Intratumoral injection of Cur injectable formulations}

Formulations of saline, single injection of free Cur, repeated injections of free Cur, Cur-M alone and Cur-M-loaded $\mathrm{CCH}$ were prepared for in vivo intratumoral injections. Each formulation was injected directly into the tumor of xenografted animals (Figure 9a). 

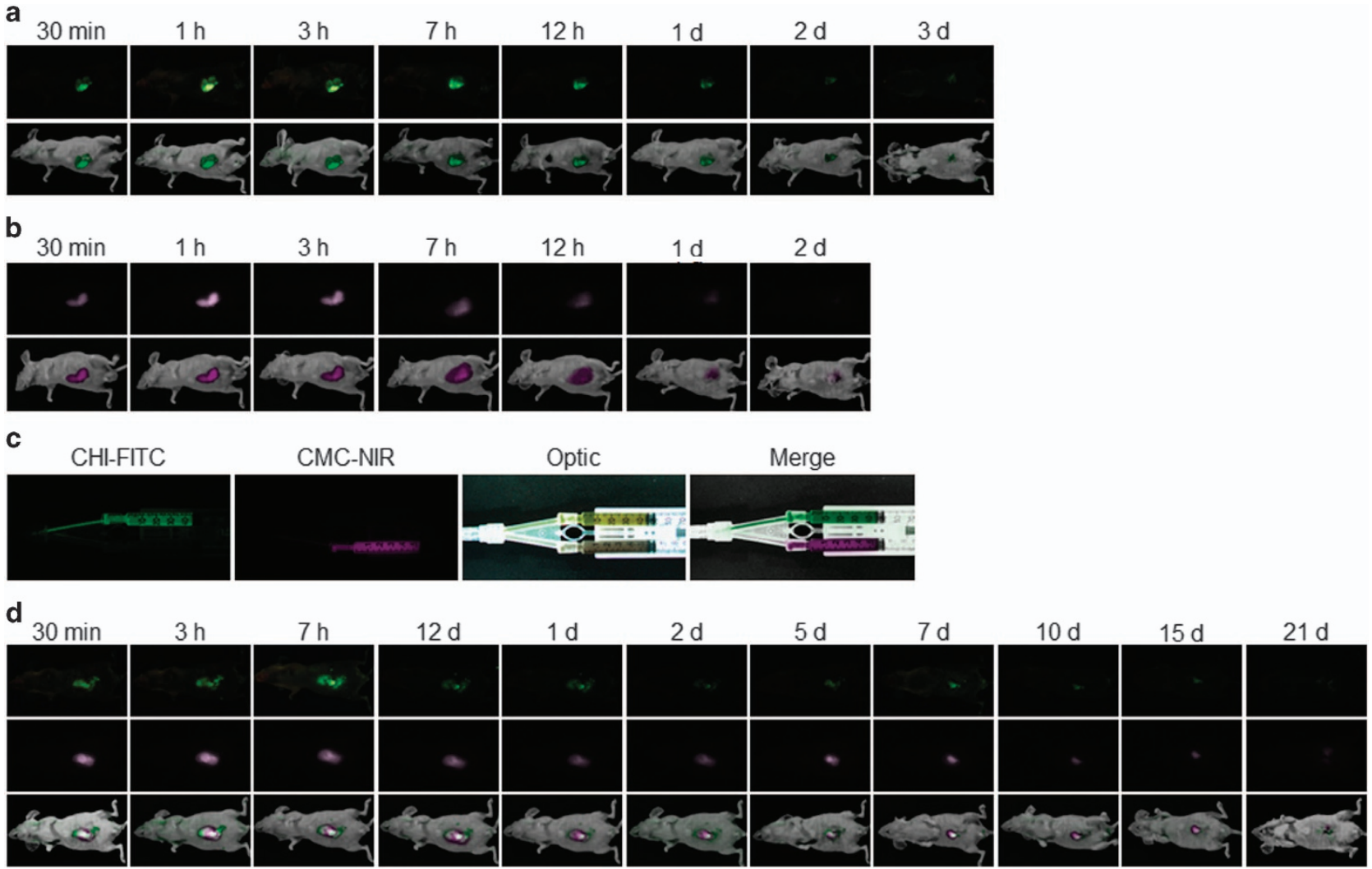

Figure 6 (a) Fluorescent images after injection of CHI-FITC over 3 days; (b) near infrared (NIR) images after injection of CMC-NIR over 2 days; (c) fluorescent image of CHI-FITC, NIR image of CMC-NIR, optical image and merged fluorescent and NIR images of CHI-FITC and CMC-NIR; (d) merged fluorescent and NIR images after injection of CHI-FITC and CMC-NIR over 21 days.

a

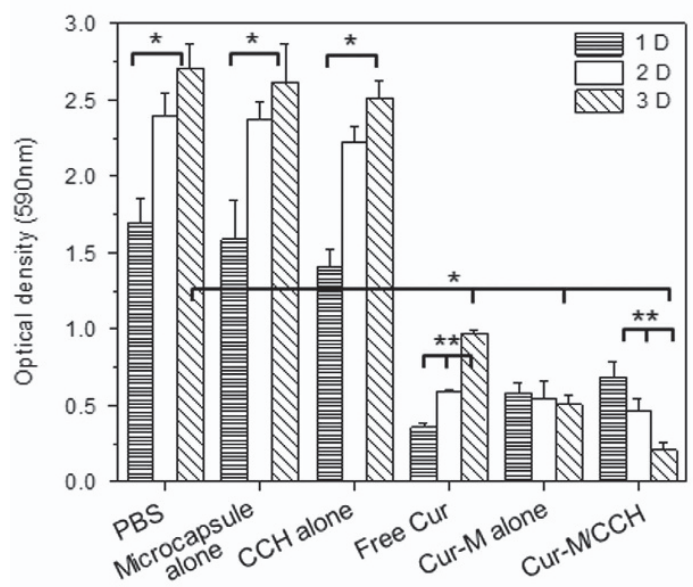

b

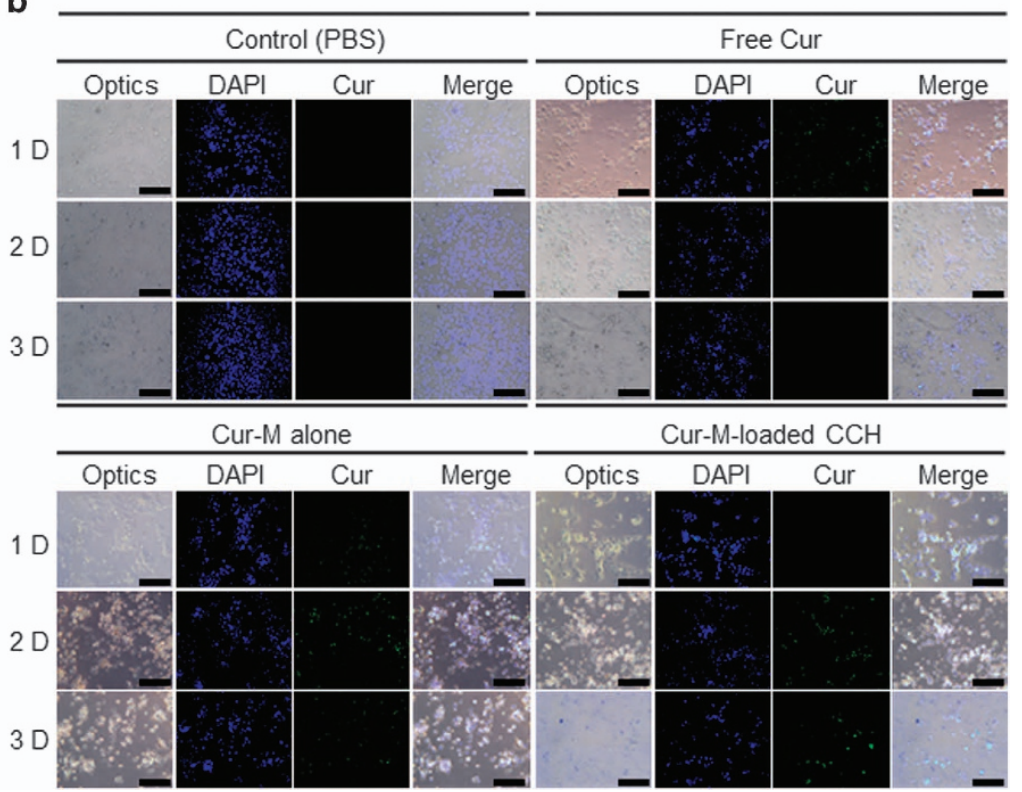

Figure 7 (a) Viability of A-253 tumor cell cells exposed to the test formulations measured with an MTT (MTT (3-(4,5-dimethylthiazol-2-yl)-2,5diphenyltetrazolium bromide) assay. Cells grown on a culture plate were used as a control group. Statistical analyses were performed using one-way analysis of variance with Bonferroni's multiple comparison test $\left({ }^{*} P<0.001\right.$ and $\left.{ }^{* *} P<0.05\right)$. (b) Optical and fluorescent images showing the morphology of A-253 cells treated with PBS, free Cur alone, Cur-M alone and Cur-M-loaded CCH at days 1, 2 and 3. Scale bars: $20 \mu \mathrm{m}$ (×200 magnification).

Antiproliferative activities of formulations were assessed by monitoring changes in the tumor volume. The average tumor size on day 0 was $110 \pm 30 \mathrm{~mm}^{3}$. The change in tumor volume was monitored for
20 days after intratumoral injection (Figures $10 \mathrm{~b}$ and c). After 20 days, the size of the tumors for saline injection, single injection of free Cur and repeated injections of free Cur reached a size of $8,6.5$ and 5.3 
a

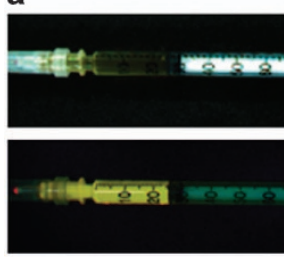

Free Cur

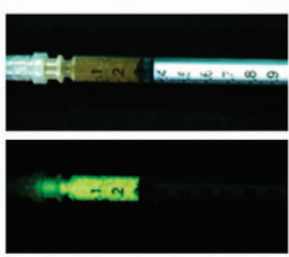

Cur-M alone

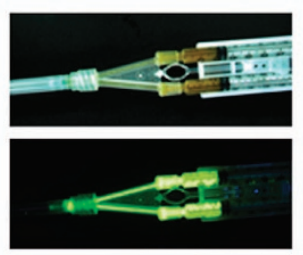

Cur-M-loaded $\mathrm{CCH}$

b Free Cur

\begin{tabular}{|c|c|c|c|c|c|c|c|}
\hline $30 \mathrm{~min}$ & $1 \mathrm{~h}$ & $3 \mathrm{~h}$ & $5 \mathrm{~h}$ & $10 \mathrm{~h}$ & $1 \mathrm{~d}$ & $2 d$ & $3 d$ \\
\hline 7 & 3 & 9 & 2. & ? & , & $\checkmark$ & 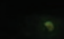 \\
\hline & & & & & & & \\
\hline
\end{tabular}

c Cur-M alone

\begin{tabular}{|c|c|c|c|c|c|c|c|c|c|c|}
\hline 30min & $1 \mathrm{~h}$ & $3 \mathrm{~h}$ & $5 \mathrm{~h}$ & $10 \mathrm{~h}$ & $1 \mathrm{~d}$ & $2 d$ & $4 d$ & $6 c$ & $8 d$ & $10 \mathrm{~d}$ \\
\hline$*$ & . & 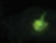 & $*$ & $*$ & 4 & 6 & $\forall$ & $\bullet$ & 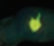 & 0 \\
\hline & & & & & & & & & & \\
\hline
\end{tabular}

d Cur-M-loaded $\mathrm{CCH}$

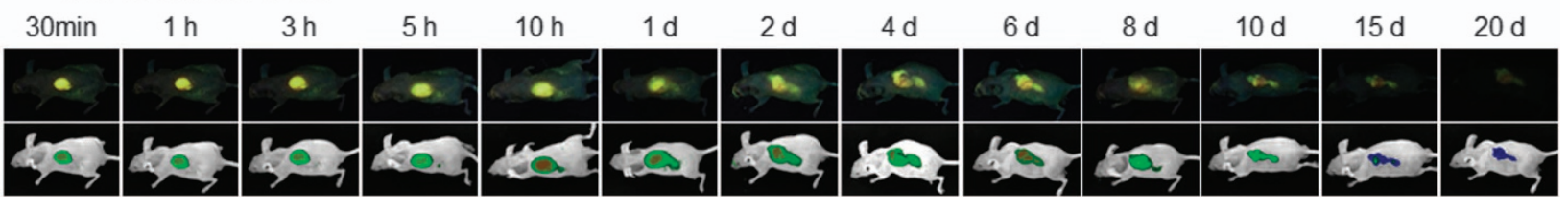

Figure 8 Fluorescent images of (a) syringes with free Cur, Cur-M and Cur-M-loaded CCH and mice subcutaneously injected with (b) free Cur, (c) Cur-M and (d) Cur-M-loaded $\mathrm{CCH}$. Imaging was performed at the indicated times after injection.

times that of the day 0 tumors, respectively. These results indicate that free Cur has antitumor activity, although the effect is small.

In contrast, Cur-M alone and Cur-M-loaded CCH suppressed tumor growth, restricting the total increase in tumor volume to $\sim 3.8$ and 2.6 times the original tumor size, respectively.

The tumor volume doubling times and tumor growth rates are summarized in Table 1. Injections of saline produced very short doubling times of 6.8 days, and a rapid rate of tumor growth of $\sim 39 \mathrm{~mm}^{3}$ per day. Single and repeated injections of free Cur extended the doubling times to 7.5 and 7.7 days, respectively, and decreased the rate of tumor growth to 33 and $29 \mathrm{~mm}^{3}$ per day, respectively. Free Cur showed antitumor activity compared to controls, but there was only a slight difference between the single and repeated injections of free Cur, probably due to its metabolism and systemic elimination.

In mice receiving a single intratumoral injection of Cur-M alone and Cur-M-loaded $\mathrm{CCH}$, the average tumor volume doubling time increased to 8.6 and 9.6 days, respectively, and the average growth rate decreased to 21 and $17 \mathrm{~mm}^{3}$ per day, respectively. In particular, the Cur-M-loaded $\mathrm{CCH}$ produced greater suppression of tumor growth than Cur-M alone. This was likely caused by the sustained Cur release from the Cur-M-loaded $\mathrm{CCH}$ due to the $\mathrm{CCH}$ hydrogel acting as a protective shell for the Cur-M.

In vivo Cur release from intratumoral injections of Cur injectable formulations

Free Cur, Cur-M and Cur-M-loaded $\mathrm{CCH}$ were injected directly into the tumor of xenografted mice to examine the persistence of in vivo
Cur release in tumors (Figure 10). Injection of free Cur, Cur-M and Cur-M-loaded $\mathrm{CCH}$ produced green fluorescent signals in the tumors at the initial $30 \mathrm{~min}$ time points.

The area of fluorescence in the mouse injected once with free Cur increased by diffusion from the injection site, with a maximal area observed $5 \mathrm{~h}$ after injection. After day 1 , negligible fluorescence was observed.

Prolonged fluorescence was observed in the mouse treated with Cur-M and Cur-M-loaded CCH. Cur-M and Cur-M-loaded CCH showed maximal fluorescence areas after 5 and $10 \mathrm{~h}$, respectively. Cur$\mathrm{M}$ fluorescence was sustained for 10 days, while that of Cur-M-loaded $\mathrm{CCH}$ was observed for more than 20 days. These results indicate that the Cur in Cur-M and Cur-M-loaded CCH could remain in the tumor for at least 10 and 20 days, respectively.

\section{Biodistribution of Cur}

To examine the biodistribution of Cur, the tumors and various organs were collected 1, 10 and 20 days after injection of saline, free Cur (single and repeated), Cur-M alone and Cur-M-loaded CCH. The amount of Cur in tumors and the intestine, stomach, lung, liver, spleen, kidney and heart was quantified (Figure 11).

One day after a single injection of free Cur, $5 \%$ of the Cur was found in the tumor, but this almost disappeared by days 10 and 20 . For the repeated injections of free Cur at days 4, 9 and 14, the amount of Cur remaining in the tumor was $7 \%$ at day 10 and $<1 \%$ at day 20 . This indicates that only a small amount of Cur could be maintained in the tumor, despite the repeated injections. Very small amounts of Cur 
a

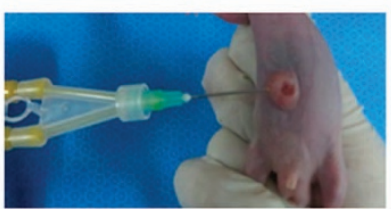

b

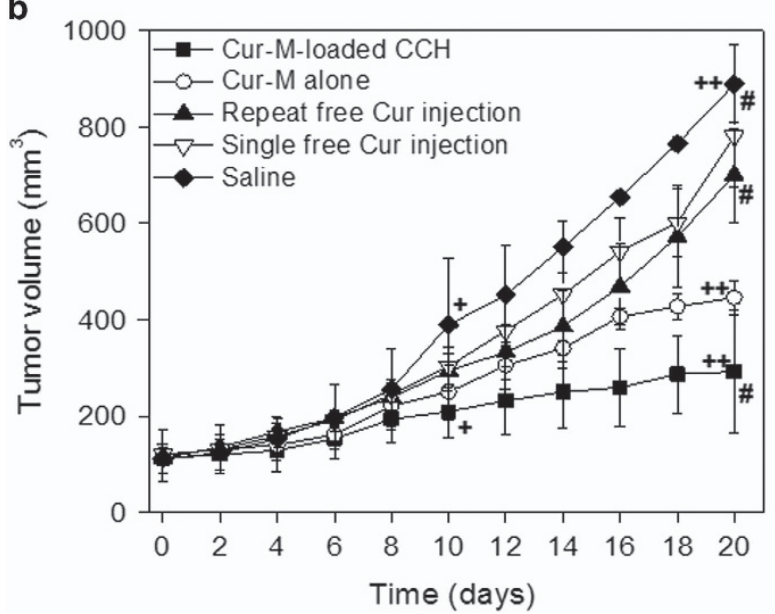

c

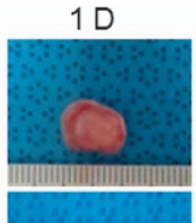

$10 \mathrm{D}$

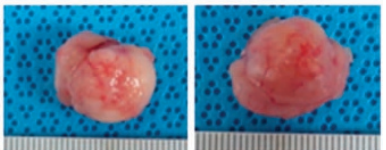

Free Cur

single

Free Cur repeat

Cur-M alone

Cur-M/CCH
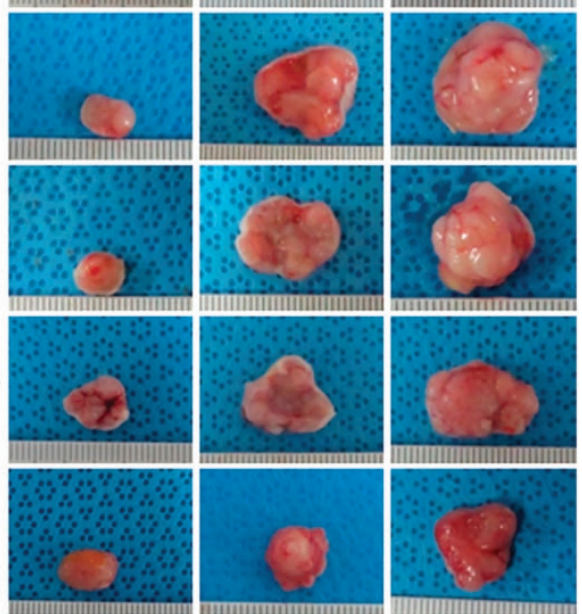

Figure 9 (a) Image of intratumoral injection to a mouse bearing an A-253 tumor cell xenograft; (b) inhibitory effects on tumor growth of saline, single injection of free Cur, repeated injections of free Cur at days 4, 9 and 14, Cur-M alone and Cur-M-loaded $\mathrm{CCH}$; (c) changes in tumor volume after intratumoral injection of saline, single injection of free Cur alone, repeated injections of free Cur alone, Cur-M alone and Cur-M-loaded $\mathrm{CCH}$. Images were taken of tumor masses excised from mice bearing A-253 tumor cell xenografts at the time of euthanasia (1, 10 and 20 days (D) post-treatment). Each solution was injected into xenograft-bearing mice after the initial tumor had reached a volume of $150-200 \mathrm{~mm}^{3}$. Statistical analyses were performed using one-way analysis of variance with Bonferroni's multiple comparison test ${ }^{++} P<0.001$ versus the saline-injected group at day $20 ;+P<0.01$ versus Cur-Mloaded $\mathrm{CCH}$-injected group at day 10; $P<0.01$ versus Cur-M-loaded $\mathrm{CCH}$-injected group at 20 days.

a Free Cur

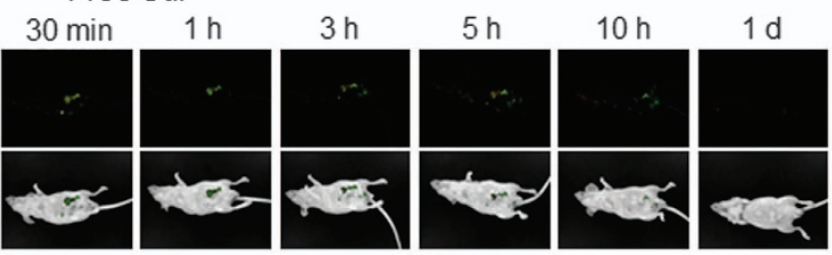

b Cur-M alone
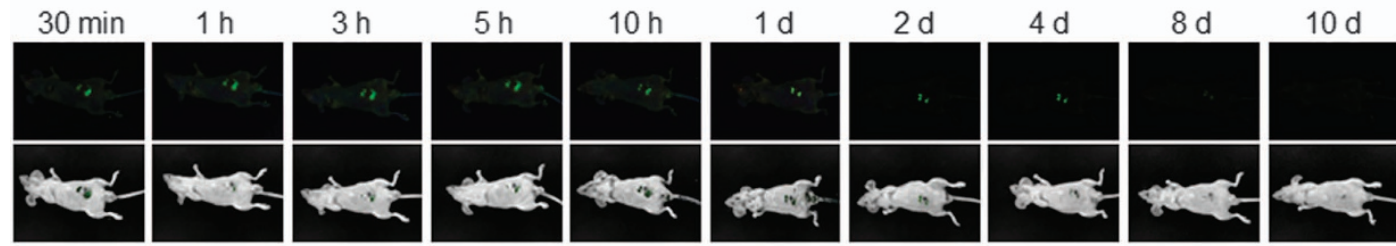

c Cur-M-loaded $\mathrm{CCH}$
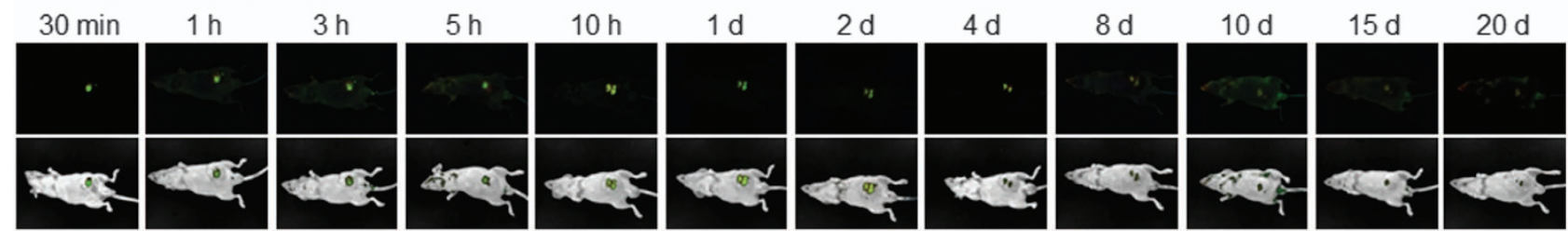

Figure 10 Fluorescent images after intratumoral injection of xenograft-bearing mice with (a) free Cur, (b) Cur-M alone, (c) Cur-M-loaded CCH as drug formulations. Images were acquired at the indicated times after drug injection. 
Table 1 Tumor volume growth rate and tumor volume doubling time after injection

\begin{tabular}{|c|c|c|c|c|c|}
\hline & Saline & Free Cur single injection & Free Cur repeated injections & Cur- $M$ alone & Cur-M-loaded CCH \\
\hline Tumor volume doubling time (days) & $6.8 \pm 1.1$ & $7.5 \pm 0.8$ & $7.7 \pm 0.8$ & $8.6 \pm 0.4$ & $9.6 \pm 2.6^{a}$ \\
\hline Tumor doubling rate ( $\mathrm{mm}^{3}$ per day) & $38.8 \pm 5.5$ & $33.0 \pm 5.7$ & $29.4 \pm 4.9$ & $20.9 \pm 5.4^{b}$ & $17.1 \pm 6.5^{b}$ \\
\hline
\end{tabular}

${ }^{\mathrm{a}} P<0.1$ and ${ }^{\mathrm{b}} P<0.05$ versus saline.

a

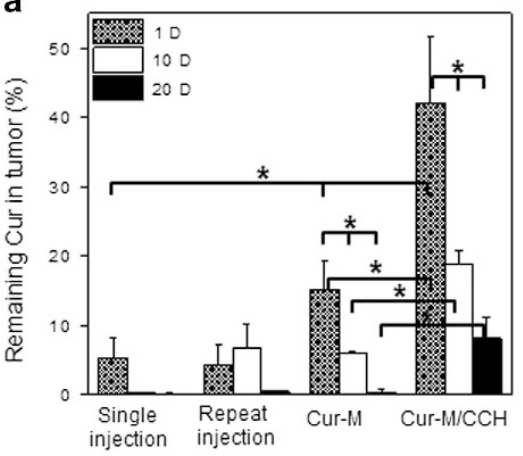

b

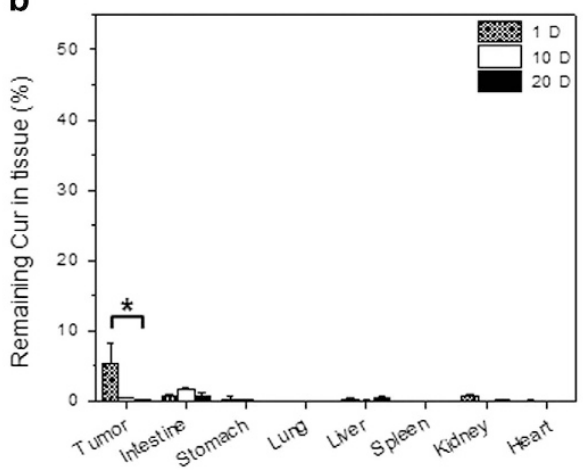

C

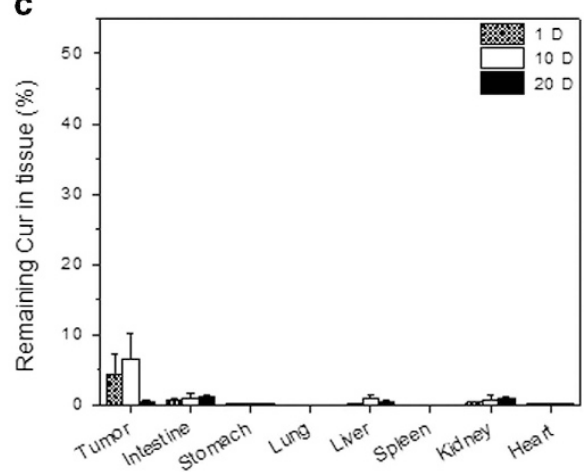

d

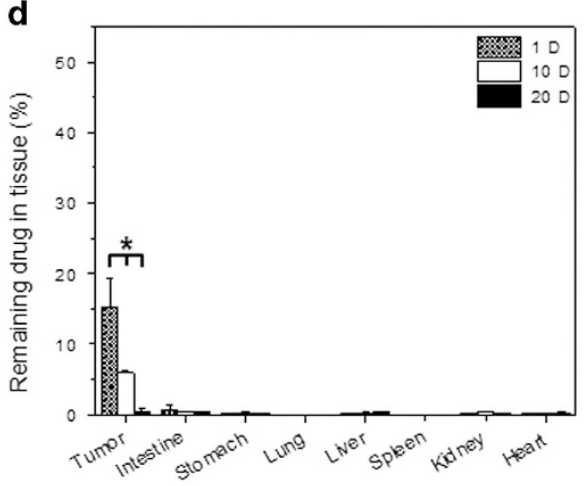

e

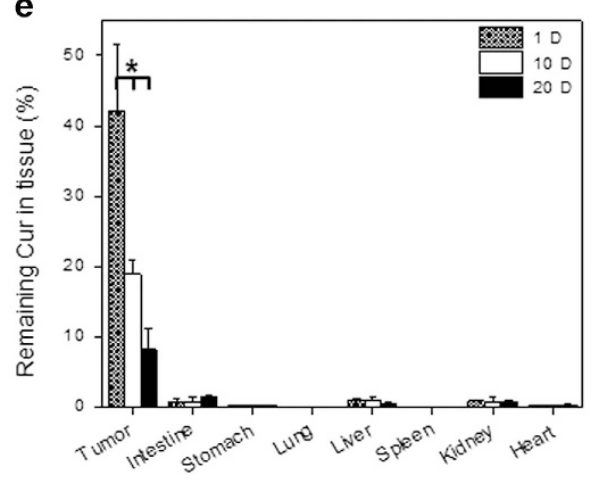

Figure 11 Persistence of Cur (a) in tumors after intratumoral injection of xenograft-bearing mice with a single injection of free Cur alone, repeated injections of free Cur alone, Cur-M alone and Cur-M-loaded $\mathrm{CCH}$ (b-e) Cur distribution in organs after intratumoral injection of (b) single injection of free Cur alone, (c) repeated injections of free Cur alone, (d) Cur-M alone and (e) Cur-M-loaded $\mathrm{CCH}\left({ }^{*} P<0.05\right)$.

$(<2 \%$ total) were found in the other organs that were collected from single and repeated injections of free Cur. This is indicative of the rapid metabolism and/or rapid systemic elimination of Cur.

In the case of Cur-M alone, Cur remained in the tumor at 15, 6 and $<1 \%$ at days 1,10 and 20, respectively, indicating a sustained release of Cur from Cur-M. Meanwhile, after injection of Cur-M-loaded $\mathrm{CCH}, 42 \%$ of Cur was observed in the tumor on day $1(P<0.05)$. Cur remained in the tumors at $19 \%$ on day 10 and $8 \%$ on day 20 $(P<0.05)$. This was probably due to sustained Cur release from the Cur-M-loaded $\mathrm{CCH}$, which was kept in the tumor for an extended period by the $\mathrm{CCH}$ hydrogel.

In addition, very small amounts of Cur ( $<3 \%$ total) were found in the other organs that were collected from the mice injected with Cur$\mathrm{M}$ alone and Cur-M-loaded $\mathrm{CCH}$.

These results indicate that direct intratumoral injection of Cur-M alone and Cur-M-loaded CCH significantly enhances the distribution of Cur in the tumor. In addition, the sustained release of Cur can be achieved by using Cur-M-loaded CCH hydrogels.

\section{Histology studies}

Histological sections of the tumors injected with saline, free Cur (single and repeated), Cur-M alone and Cur-M-loaded CCH on days
1, 10 and 20 were examined after staining with hematoxylin and eosin (Supplementary Figure S5).

After intratumoral injection of saline, little necrosis was apparent. The number of blood vessels increased as the implantation time increased. Tumors injected with free Cur (single and repeated) exhibited a few regions with some degree of necrotic indices, compared to saline treated tumors. This implies that free Cur can exhibit some antitumor activity.

Tumors injected with Cur-M alone and Cur-M-loaded $\mathrm{CCH}$ exhibited higher necrosis. The necrotic regions that were interspersed between areas of viable tumor increased as implantation time increased. In addition, small blood vessels were observed in tumors injected with Cur-M alone and Cur-M-loaded CCH. Cur-M alone and Cur-M-loaded $\mathrm{CCH}$ persisted at the injected site for the full experimental period of 20 days.

Figure 12a images show blue 4',6-diamidino-2-phenylindole fluorescence (nuclei), red TUNEL fluorescence (apoptosis), green Cur fluorescence and a merger of the three images for tumors treated with saline, free Cur (single and repeated), Cur-M alone and Cur-M-loaded $\mathrm{CCH}$ after 1,10 and 20 days.

In the tumors treated with saline, bright blue fluorescence, corresponding to the nuclei of live cells was evident, and red 
a

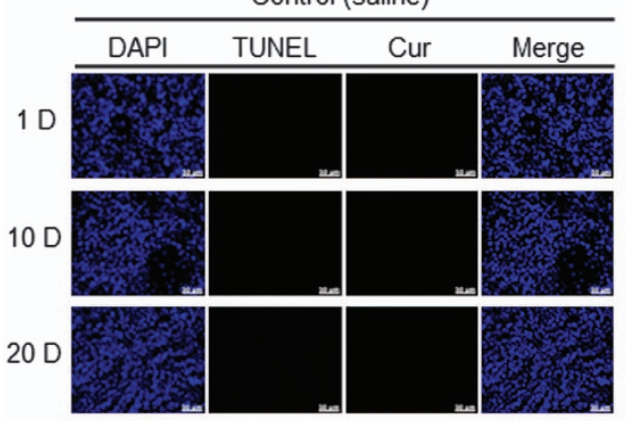

Cur-M alone

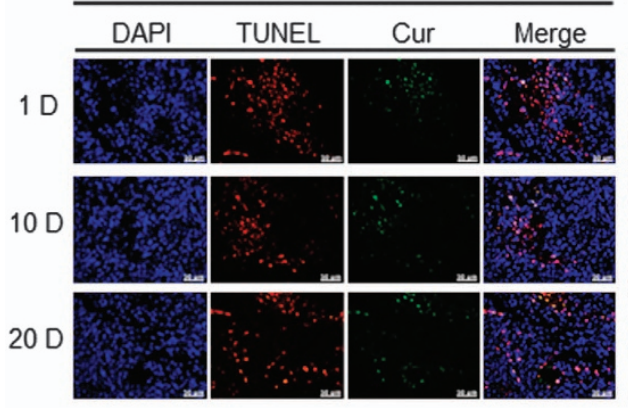

Free Cur single injection

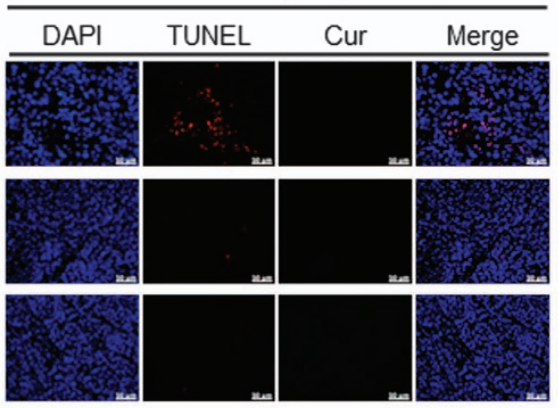

Cur-M-loaded CCH

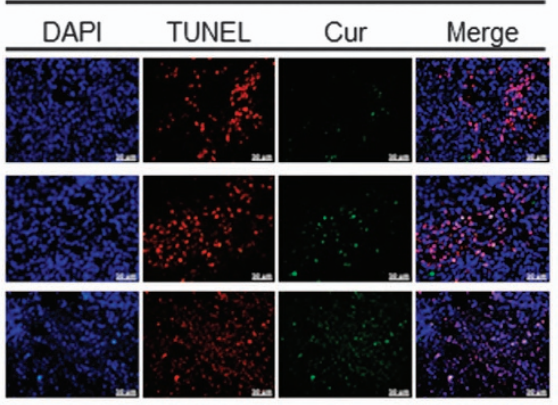

Free Cur repeated injection

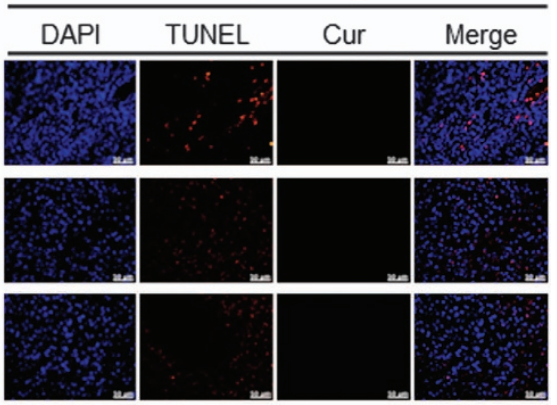

b

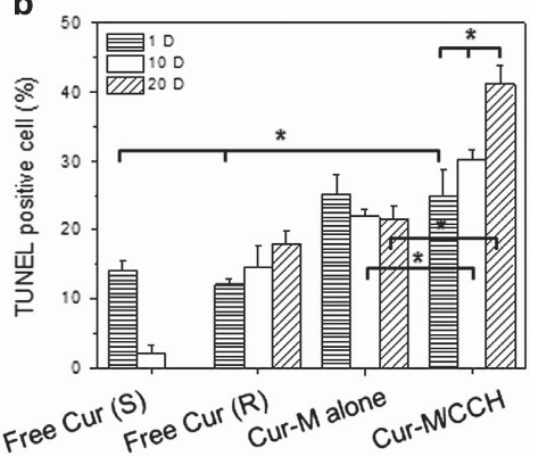

Figure 12 (a) 4',6-Diamidino-2-phenylindole (DAPI) staining (blue, nuclei), TUNEL staining (green, apoptotic cells), Cur (green) and merged images of tumors on days (D) 1, 10 and 20 after intratumoral injection of xenograft-bearing mice with a single injection of free Cur alone, repeated injections of free Cur alone, Cur-M alone and Cur-M-loaded CCH ( $\times 200$ magnification; scale bar: $50 \mu \mathrm{m})$. (b) Number of TUNEL-positive cells in images from tumors injected with a single injection of free Cur alone, repeated injections of free Cur alone, Cur-M alone and Cur-M-loaded $\mathrm{CCH}\left({ }^{*} P<0.05\right)$.

fluorescence from TUNEL staining was absent. For the tumors treated with a single injection of free Cur, the green fluorescence of Cur was present at day 1 and then disappeared at day 10. The red fluorescence attributable to apoptotic cells gradually decreased as the implantation time increased. Meanwhile, for the tumors treated with repeated injections of free Cur, the green fluorescence of Cur was maintained for 1, 10 and 20 days. Red TUNEL fluorescence was also present at all time points. The merger of blue, green and red fluorescence produced bright white areas, indicating areas of overlap that strongly suggest tumor cell apoptosis by the injected Cur.

The tumors treated with Cur-M alone showed distinct red fluorescence due to tumor cell apoptosis and green fluorescence due to the Cur released from Cur-M. Considerable degrees of red fluorescence from TUNEL staining were detected in tumors injected with Cur-M-loaded $\mathrm{CCH}$, indicating large numbers of apoptotic cells. The merged white areas of overlapping fluorescence indicate that the Cur released from Cur-M-loaded $\mathrm{CCH}$ can induce apoptosis of the tumor cells.

TUNEL-positive cells were counted and normalized to the total stained tissue area in order to determine the extent of apoptosis (Figure 12b). The percentage of apoptotic cells (TUNEL-positive cells) in the group treated with single injections of free Cur was $14 \%$ at day 1 and decreased to $<1 \%$ as the implantation time increased. For the repeated injections of free Cur, the percentage increased to $12 \%$ at day $1,15 \%$ at day 10 and $18 \%$ at day 20 , as the implantation time increased. The apoptotic cells in the group treated with Cur-M alone was $27 \%$ at day 1 and was maintained at $\sim 22 \%$ as the implantation time increased, reflecting the sustained release of Cur from Cur-M. In the case of intratumoral injections of Cur-M-loaded $\mathrm{CCH}$, the apoptosis was $27 \%$ at day $1,31 \%$ at 10 days and $42 \%$ at day 20 .
This finding indicates that the sustained release of Cur from Cur-Mloaded $\mathrm{CCH}$ can cause enhanced apoptosis of the tumor cells.

\section{DISCUSSION}

Various naturally derived and synthetic biomaterials have been successfully used for manufacturing of drug depots. In the present work, we sought to discover an electrostatic, cross-linkable drug depot. The dynamics of electrostatic, cross-linkable hydrogels allow injection before mixing and solidification after mixing, allowing for a minimally invasive drug depot.

The electrostatic cross-linking between the CMC and CHI solutions substantially changed the viscosity of the solutions. The viscosity varied, with a sharp maximum at equal ratios of $\mathrm{CMC}$ and $\mathrm{CHI}$ solution, and then dropping at unequal ratios, likely reflecting changes in the electrostatic interactions between CMC and CHI. The electrostatic, cross-linked $\mathrm{CCH}$ depot was gelatinized by the electrostatic interaction between the carboxylic groups of the CMC chains and the amine groups of the CHI.

The electrostatic, cross-linked $\mathrm{CCH}$ depot did not flow upon inversion of its vial and revealed an interconnected porous network structure that allowed Cur drug diffusion. The $\mathrm{CCH}$ depots persisted for an extended period in vivo, but did diminish with implantation time, probably due to dissipation of CMC and $\mathrm{CHI}$ by breaking of electrostatic interactions or competition of electrostatic interactions with biological ionic compounds. Collectively, in vitro and in vivo, we found that $\mathrm{CMC}$ and $\mathrm{CHI}$ solutions successfully formed a $\mathrm{CCH}$ depot, providing support for our hypothesis that an electrostatic, cross-linked $\mathrm{CCH}$ depot can be used as a Cur depot.

In the clinical management of cancer, cancer patients can have a tumor surgically removed as much as possible, followed by the 
administration of local radiotherapy and/or systemic chemotherapeutic drugs to prevent tumor recurrence.

A naturally occurring, yellow-colored polyphenol, Cur, can be utilized as a potent anticancer drug. It inhibits proliferation and induces apoptosis in various cancer cells. Cur-based preclinical studies and clinical trials have been conducted in animal models and cancer patients. The trials conducted thus far have indicated the therapeutic potential of Cur against cancers. However, the clinical applications of Cur are limited due to its rapid metabolism under biological conditions, leading to low efficacy due to insufficient exposure of cancer cells in the tumor to the Cur.

Thus, we attempted to overcome these limitations and drawbacks by direct intratumoral injection of a sustained Cur delivery system, because the bioavailability and therapeutic efficacy of Cur can be increased by extending its release within the tumor over a longer period. In addition, few studies have examined injectable, electrostatic, cross-linkable formulations for intratumoral injection of Cur depots.

In previous studies, our group reported the manufacture of injectable drug depots, using injectable hydrogels. ${ }^{3,26-30}$ In the current study, we designed injectable formulations of Cur-M alone and Cur-M dispersed inside an outer $\mathrm{CCH}$ shell. We confirmed that Cur-M was easily incorporated in the injectable $\mathrm{CMC}$ and $\mathrm{CHI}$ formulation. The formulations of Cur-M or Cur-M-loaded $\mathrm{CCH}$ were successfully injected into the centers of tumors, using a 21-gauge needle, to produce Cur depots in a minimally invasive manner.

Importantly, Cur-M or Cur-M-loaded $\mathrm{CCH}$ injectable formulations are easily handled, easily injected, easily amended in their formulation and have good structural integrity in vivo. Collectively, these suggest that the formulations are well suited for clinical use as promising candidates for Cur depots.

The availability of Cur at the target site was maintained for extended periods, which was confirmed by fluorescence imaging techniques, while single and repeated injections of free Cur led to faster Cur disappearance. We, therefore, confirmed that the injectable Cur-M or Cur-M-loaded $\mathrm{CCH}$ formulations designed in this study act as Cur depots that produce sustained Cur release inside the tumor.

The Cur-M or Cur-M-loaded $\mathrm{CCH}$ formulations led to almost complete cell death as a function of culture time in vitro. The Cur-Mloaded $\mathrm{CCH}$ formulation inhibited in vivo tumor growth more effectively than Cur-M alone. The in vivo Cur biodistribution data showed that high Cur concentrations were maintained only in the target tumor, with little distribution of Cur to normal tissues.

Cur degrades quickly at normal physiological condition. ${ }^{14-16,31-33}$ In this work, the amount of Cur remaining in the tumor after intratumoral injection of the free Cur was only $5 \%$ at 1 day in tumor. This result was in accordance to previous reports where it has been observed that Cur decomposed above 90\% within 1 day at physiological condition. ${ }^{31-33}$ Thus, we conjecture that low Cur amount is due to the rapid metabolic degradation and/or rapid systemic elimination of Cur from tumor.

Meanwhile, intratumoral injection of Cur-M alone showed $15 \%$ Cur remaining in the tumor at 1 day indicating the stability increasing of Cur and then a sustained release of Cur from Cur-M for 10 days in tumor. Cur-M alone was longer than the maintenance time of free Cur in the tumor.

The tumor after injection of Cur-M-loaded CCH contained $~ 40 \%$ of Cur at 1 day. This quantity was higher than that in the tumor after intratumoral injection of Cur-M. Importantly Cur-M-loaded $\mathrm{CCH}$ showed prolonged release of Cur for at least 20 days. This result indicated that Cur-M-loaded $\mathrm{CCH}$ was contributed in preventing the rapid metabolic degradation and/or rapid systemic elimination of Cur from tumor.

In this work, we confirmed that the injectable, electrostatic, crosslinkable Cur formulations were able to establish Cur depots in tumors for extended periods, and then evaluated whether the electrostatic, cross-linked Cur depot produced synergistically enhanced tumor suppression after intratumoral injection. Collectively, Cur caused significant antitumor activity via sufficient exposure of the cancer cells to Cur and maintaining Cur therapeutic concentrations.

\section{CONCLUSION}

CMC and $\mathrm{CHI}$ solutions successfully formed an electrostatic, crosslinked $\mathrm{CCH}$ depot and maintained this structure for an extended period. Based on the results of the intratumoral injection experiments, it is reasonable to conclude that Cur-M or Cur-M-loaded $\mathrm{CCH}$ formulations could induce long-lasting Cur release and significantly inhibit tumor growth in vivo. The long-lasting release of Cur by the $\mathrm{CCH}$ hydrogel acting as a protective outer shell in the Cur-M-loaded $\mathrm{CCH}$ depot led to greater inhibition of tumor growth.

\section{CONFLICT OF INTEREST}

The authors declare no conflict of interest.

\section{ACKNOWLEDGEMENTS}

This study was supported by a grant from a Basic Science Research Program (2016R1A2B3007448) and Priority Research Centers Program (2010-0028294) through the National Research Foundation of Korea (NRF) funded by the Ministry of Education.

Author contributions: DYK, SHP, PP, JYH and HYL prepared materials carried out experiments as well as animal experiments. DYK, SHP, JHK and MSK discussed the rheology. DYK, SHP, PP, JYH, HYL, BHM and MSK prepared and discussed the stained images. MSK designed all experiments and wrote the paper. All authors discussed the results and commented on the manuscript.

1 Kim, D. Y., Kwon, D. Y., Kwon, J. S., Kim, J. H., Min, B. H. \& Kim, M. S. Injectable in situ-forming hydrogels for regenerative medicines. Polym. Rev. 55, 407-452 (2015).

2 Das, S., Zhou, K., Ghosh, D., Jha, N. N., Singh, P. K., Jacob, R. S., Bernard, C. C., Finkelstein, D. I., Forsythe, J. S. \& Maji, S. K. Implantable amyloid hydrogels for promoting stem cell differentiation to neurons. NPG Asia Mater. 8, e304 (2016)

3 Wang, J., Zhang, F., Tsang, W. P., Wan, C. \& Wu, C. Fabrication of injectable high strength hydrogel based on 4-arm star PEG for cartilage tissue engineering. Biomaterials 120, 11-21 (2017).

4 Kim, K. S., Park, J. H., Park, S. H., Lee, H. Y., Kim, J. H. \& Kim, M. S. An injectable, click-cross-linked small intestinal submucosa drug depot for the treatment of rheumatoid arthritis. Adv. Healthc. Mater. 5, 3105-3117 (2016).

5 Lee, J. Y., Kang, Y. M., Kim, E. S., Kang, M. L., Lee, B., Kim, J. H., Min, B. H., Park, K. N. \& Kim, M. S. In vitro and in vivo release of albumin from an electrostatically crosslinked in situ-forming gel. J. Mater. Chem. 20, 3265-3271 (2010).

6 Kamata, H., Li, X., Chung, U. I. \& Sakai, T. Design of hydrogels for biomedical applications. Adv. Healthc. Mater. 4, 2360-2374 (2015).

7 Whang, M. \& Kim, J. Synthetic hydrogels with stiffness gradients for durotaxis study and tissue engineering scaffolds. Tissue Eng. Reg. Med. 13, 126-139 (2016).

8 Xu, H. L., Tian, F. R., Lu, C. T., Xu, J., Fan, Z. L., Yang, J. J., Chen, P. P., Huang, Y. D., Xiao, J. \& Zhao, Y. Z. Thermo-sensitive hydrogels combined with decellularised matrix deliver bFGF for the functional recovery of rats after a spinal cord injury. Sci. Rep. 6, 38332 (2016).

9 Arancibia, C., Bayarri, S. \& Costell, E. Comparing carboxymethyl cellulose and starch as thickeners in oil/water emulsions. Implications on rheological and structural properties. Food Biophys. 8, 122-136 (2013).

10 Du, W. L., Niu, S. S., Xu, Y. L., Xu, Z. R. \& Fan, C. L. Antibacterial activity of chitosan tripolyphosphate nanoparticles loaded with various metal ions. Carbohydr. Polym. 75, 385-389 (2009).

11 Lv, Q. Y., Tao, Y. X., Qin, Y., Chen, C. X., Pan, Y., Deng, L. H., Liu, L. \& Kong, Y. Highly fluorescent and morphology-controllable graphene quantum dots-chitosan hybrid xerogels for in vivo imaging and $\mathrm{pH}$-sensitive drug carrier. Mater. Sci. Eng. C 67 , 478-485 (2016). 
12 Canal, P., Chatelut, E. \& Guichard, S. Practical treatment guide for dose individualization in cancer chemotherapy. Drugs 56, 1019-1038 (1998).

13 Lelli, D., Sahebkar, A., Johnston, T. P. \& Pedone, C. Curcumin use in pulmonary diseases: state of the art and future perspectives. Pharmacol. Res. 115, 133-148 (2017).

14 Deng, Y. I., Verron, E. \& Rohanizadeh, R. Molecular mechanisms of anti-metastatic activity of curcumin. Anticancer Res. 36, 5639-5647 (2016).

15 Lee, W. H., Loo, C. Y., Young, P. M., Traini, D., Mason, R. S. \& Rohanizadeh, R. Recent advances in curcumin nanoformulation for cancer therapy. Expert Opin. Drug Deliv. 11, 1183-1201 (2014).

16 Bansal, S. S., Goel, M., Aqil, F., Vadhanam, M. V. \& Gupta, R. C. Advanced drug delivery systems of curcumin for cancer chemoprevention. Cancer Prev. Res. (Phila) 4, 1158-1171 (2011).

17 Rathore, N. \& Rajan, R. S. Current perspectives on stability of protein drug products during formulation, fill and finish operations. Biotechnol. Prog. 24, 504-514 (2008).

18 Long, Y., Liu, C. Y., Zhao, B., Song, K., Yang, G. Q. \& Tung, C. H. Bio-inspired controlled release through compression-relaxation cycles of microcapsules. NPG Asia Mater. 7, e148 (2015).

19 Kim, B. S., Oh, J. M., Hyun, H., Kim, K. S., Lee, S. H., Kim, Y. H., Park, K. N., Lee, H. B. \& Kim, M. S. Insulin-loaded microcapsules for in vivo delivery. Mol. Pharm. 6, 353-365 (2009).

20 Fakhari, A. \& Subramony, J. A. Engineered in-situ depot-forming hydrogels for intratumoral drug delivery. J. Control Release 220, 465-475 (2015).

21 Kim, D. Y., Kwon, D. Y., Kwon, J. S., Park, J. H., Park, S. H., Oh, H. J., Kim, J. H., Min, B. H., Park, K. N. \& Kim, M. S. Synergistic anti-tumor activity through combinational intratumoral injection of an in-situ injectable drug depot. Biomaterials 85, 232-245 (2016).

22 Seo, H. W., Kim, D. Y., Kwon, D. Y., Kwon, J. S., Jin, L. M., Lee, B., Kim, J. H., Min, B. H. \& Kim, M. S. Injectable intratumoral hydrogel as 5-fluorouracil drug depot. Biomaterials 34, 2748-2757 (2013).

23 Cho, J. K., Kuh, H. J. \& Song, S. C. Injectable poly(organophosphazene) hydrogel system for effective paclitaxel and doxorubicin combination therapy. J. Drug Target 22, 761-767 (2014).

24 Kim, J. I., Lee, B. S., Chun, C., Cho, J. K., Kim, S. Y. \& Song, S. C. Long-term theranostic hydrogel system for solid tumors. Biomaterials 33, 2251-2259 (2012).

25 Chen, S., Hirota, N., Okuda, M., Takeguchi, M., Kobayashi, H., Hanagata, N. \& Ikoma, T. Microstructures and rheological properties of tilapia fish-scale collagen hydrogels with aligned fibrils fabricated under magnetic fields. Acta Biomater. 7, 644-652 (2011).

26 Jang, J. Y., Park, S. H., Park, J. H., Lee, B. K., Yun, J. H., Lee, B., Kim, J. H., Min, B. H. \& Kim, M. S. In vivo osteogenic differentiation of human dental pulp stem cells embedded in an injectable in vivo-forming hydrogel. Macromol. Biosci. 16, 1158-1169 (2016).
27 Son, A. R., Kim, D. Y., Park, S. H., Jang, J. Y., Kim, K. S., Kim, B. J., Yin, X. Y., Kim, J. H., Min, B. H., Han, D. K. \& Kim, M. S. Direct chemotherapeutic dual drug delivery through intra-articular injection for synergistic enhancement of rheumatoid arthritis treatment. Sci. Rep. 5, 14713 (2015).

28 Kwon, J. S., Kim, S. W., Kwon, D. Y., Park, S. H., Son, A. R., Kim, J. H. \& Kim, M. S. In vivo osteogenic differentiation of human turbinate mesenchymal stem cells in an injectable in situ-forming hydrogel. Biomaterials 35, 5327-5346 (2014).

29 Kwon, J. S., Yoon, S. M., Kwon, D. Y., Kim, D. Y., Tai, G. Z., Jin, L. M., Song, B. R., Lee, B., Kim, J. H., Han, D. K., Min, B. H. \& Kim, M. S. Injectable in situ-forming hydrogel for cartilage tissue engineering. J. Mater. Chem. B 1, 3314-3321 (2013).

30 Kang, Y. M., Kim, G. H., Kim, J. I., Kim, D. Y., Lee, B. N., Yoon, S. M., Kim, J. H. \& Kim, M. S. In vivo efficacy of an intratumorally injected in situ-forming doxorubicin/poly (ethylene glycol)-b-polycaprolactone diblock copolymer. Biomaterials 32, 4556-4564 (2011).

31 Wang, Y. J., Pan, M. H., Cheng, A. L., Lin, L. I., Ho, Y. S., Hsieh, C. Y. \& Lin, J. K. Stability of curcumin in buffer solutions and characterization of its degradation products. J. Pharm. Biomed. Anal. 15, 1867-1876 (1997).

32 Blanco-Garcia, E., Otero-Espinar, F. J., Blanco-Mendez, J., Leiro-Vidal, J. M. \& Luzardo-Alvarez, A. Development and characterization of anti-inflammatory activity of curcumin-loaded biodegradable microspheres with potential use in intestinal inflammatory disorders. Int. J. Pharm. 518, 86-104 (2017).

33 Yuan, R., Zheng, F., Zhong, S., Tao, X., Zhang, Y., Gao, F., Yao, F., Chen, J., Chen, Y. \& Shi, G. Self-assembled nanoparticles of glycyrrhetic acid-modified pullulan as a novel carrier of curcumin. Molecules 19, 13305-13318 (2014).

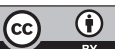

This work is licensed under a Creative Commons Attribution 4.0 International License. The images or other third party material in this article are included in the article's Creative Commons license, unless indicated otherwise in the credit line; if the material is not included under the Creative Commons license, users will need to obtain permission from the license holder to reproduce the material. To view a copy of this license, visit http:// creativecommons.org/licenses/by/4.0/

(C) The Author(s) 2017

Supplementary Information accompanies the paper on the NPG Asia Materials website (http://www.nature.com/am) 